\title{
Senolytic activity of small molecular polyphenols from olive restores chondrocyte redifferentiation and cartilage regeneration in osteoarthritis
}

Marta Varela-Eirín ${ }^{1}$, Adrián Varela-Vázquez ${ }^{1}$, Carlos Luis Paíno², Antonio CasadoDíaz $^{3}$, Alfonso Calañas Continente ${ }^{3}$, Virginia Mato ${ }^{4}$, Eduardo Fonseca ${ }^{1}$, Mustapha Kandouz $^{5}$, Alfonso Blanco ${ }^{6}$, José Ramón Caeiro ${ }^{7}$ \& María D. Mayán ${ }^{*}$

${ }^{1}$ CellCOM Research Group, Instituto de Investigación Biomédica de A Coruña (INIBIC), Servizo Galego de Saúde (SERGAS), Universidade da Coruña (UDC), Xubias de Arriba, 84, 15006 A Coruña, Spain

${ }^{2}$ Service of Neurobiology Research, Ramón y Cajal University Hospital (IRYCIS), Madrid, Spain

${ }^{3}$ UGC Endocrinology and Nutrition, Maimónides Biomedical Research Institute of Córdoba (IMIBIC), Hospital Universitario Reina Sofía - CIBERFES, Universidad de Córdoba, Spain

${ }^{4}$ Centre for Medical Informatics and Radiological Diagnosis, Universidade da Coruña, A Coruña, Spain

${ }^{5}$ Department of Pathology, School of Medicine, Wayne State University, Detroit, USA.

${ }^{6}$ Flow Cytometry Core Technologies, UCD Conway Institute, University College Dublin, Ireland.

${ }^{7}$ Department of Orthopaedic Surgery and Traumatology, Complexo Hospitalario Universitario de Santiago de Compostela (CHUS), Universidade de Santiago de Compostela (USC), Choupana s/n, 15706 Santiago de Compostela, Spain.

${ }^{*}$ Address correspondence to: Maria D. Mayan, CellCOM Research Group, Instituto de Investigación Biomédica de A Coruña (INIBIC), Servizo Galego de Saúde (SERGAS), Universidade da Coruña (UDC), Xubias de Arriba 84, 15006 A Coruña, Spain. Phone: 34-981-176395. Fax: 34-981-176398. Email: Ma.Dolores.Mayan.Santos@sergas.es

Running title: Pharmacological effect of olive-derived polyphenols in osteoarthritis.

Keywords: senescence, dedifferentiation, osteoarthritis, connexin43, cartilage, tissue regeneration, small polyphenols, oleuropein, wound healing.

Conflict of interest. The authors have declared that no conflict of interest exists.

Abbreviations: ACAN, aggrecan; AM, adipogenic medium; CBX, carbenoxolone; CD105, endoglin; CD166, CD166 antigen (ALCAM); CM, chondrogenic medium; $\mathrm{CMT}$, chondrocyte-to-mesenchymal transition; Col2A1, collagen type II; COX-2, cyclooxygenase-2; Cx43, connexin43; CxREs, connexin-response elements; ECM, extracellular matrix; EMT, epithelial-to-mesenchymal transition; FDG, fluorescein-diD-galactopyranoside; GJs, gap junctions; GJIC, gap junction intercellular communication; hMSCs, human mesenchymal stem cells; IL-1 $\beta$, interleukin 1 beta; IL-6, interleukin 6; LY, Lucifer yellow; MMP-3, matrix metalloproteinase 3; NF-kB, 
nuclear factor kappa-light-chain-enhancer of activated B cells; OA, osteoarthritis; OACs, osteoarthritic chondrocytes; OE, olive extract; OM, osteogenic medium; OSTCN, osteocalcin; p16, cyclin-dependent kinase inhibitor 2A; p53, cellular tumour antigen p53; PPARy, peroxisome proliferator-activated receptor gamma; RT-qPCR, real-time quantitative polymerase chain reaction; $S A \beta G$, senescence-associated $\beta$ galactosidase activity; SASP, senescence-associated secretory phenotype; TNFa, tumour necrosis factor alpha; Twist-1, twist-related protein 1. 


\section{Abstract}

Osteoarthritis $(O A)$ is the most prevalent disorder of articulating joints and a leading cause of disability in humans, affecting half of the world's population aged 65 years or older. Articular cartilage and synovial tissue from OA patients show an overactivity of the membrane channel protein connexin43 (Cx43) and accumulation of senescent cells associated with disrupted tissue regeneration. We have recently demonstrated the use of the $\mathrm{Cx} 43$ as an appropriate therapeutic target to halt OA progression by decreasing the accumulation of senescent cells and by triggering redifferentiation of osteoarthritic chondrocytes (OACs) into a more differentiated state, restoring the fully mature phenotype and cartilage regeneration. In this study we have found that small molecular polyphenols derived by olive extracts target $\mathrm{Cx} 43$ and senescence in OACs, synovial and bone cells from patients and in human mesenchymal stem cells (hMSCs). Our results indicate that these small molecules including oleuropein regulate the promoter activity of $\mathrm{C} x 43$ gene. The downregulation of $\mathrm{Cx} 43$ expression by oleuropein reduce gap junction intercellular communication, cellular senescence in chondrocytes and enhance the propensity of hMSCs to differentiate into chondrocytes and bone cells, reducing adipogenesis. In concordance with these results, these small molecules reduce $\mathrm{Cx} 43$ and decrease Twist-1 activity leading to redifferentiation of OACs, which restores the synthesis of cartilage ECM components (Col2A1 and proteoglycans) and reduces inflammatory and catabolic factors IL-1B, IL-6, COX-2 and MMP-3 and cellular senescence orchestrated by p53/p21 together with the synthesis of SASP via NF-kB. Altogether, our results demonstrate the use of the olive-derived polyphenols such as oleuropein as potentially effective therapeutic agents to enhance the efficacy of hMSC therapy and to induce a pro-regenerative environment in $\mathrm{OA}$ patients by restoring cellular phenotype and clearing out senescent cells in joint tissues in order to stop or prevent the progression of the disease. 


\section{Introduction.}

Osteoarthritis $(\mathrm{OA})$ is the most common degenerative joint disorder characterised by progressive degeneration of synovial joints that lead to limited mobility and pain. OA is the major cause of disability with a major socio-economic impact, which affects an increasing number of the ageing population ${ }^{1}$. Cartilage damage is the most typical sign of OA. Yet, this condition also affects the whole joint including bone, synovium, muscles, tendons and ligaments ${ }^{2}$. Articular cartilage from patients with OA shows an accumulation of dedifferentiated and senescent cells ${ }^{3-6}$ together with an increase in the production of pro-inflammatory cytokines such as IL-1B and catabolic enzymes (matrix metalloproteinases (MMPs) and aggrecanases) that lead to the breakdown of cartilage extracellular matrix (ECM) ${ }^{7}$. The prevalence of $O A$ is rising worldwide ${ }^{8}$ and the aetiology is still under study, with new insights into molecular mechanisms involved in OA progression beginning to open new possibilities to restore phenotypic stability of articular chondrocytes and to develop new approaches in order to promote cartilage repair and restore normal joint function in OA patients ${ }^{9-12}$.

Interestingly and consistent with other wound-healing disorders, osteoarthritic cartilage and synovial tissue from OA patients contain high levels of the gap junction protein connexin43 (Cx43). Therapeutic approaches targeting $\mathrm{Cx} 43$ are showing promise in benefiting several age-related and chronic degenerative diseases by modulating tissue regeneration, inflammation and response to injury ${ }^{13,14}$. Cx43 is linked to regeneration and response to injury through several different mechanisms, acting as a brake in wound healing in skin $^{14,15}$, cartilage ${ }^{4,16,17}$ and neurodegenerative disorders such as Alzheimer's disease ${ }^{18}$. Cx43 belongs to the integral membrane protein family called connexins which form hemichannels and gap junctions (GJs) that enable direct communication between neighbouring cells by interchanging electrical, metabolic and signalling molecules such as IP3, cAMP, ions, glutathione or siRNAs ${ }^{19,20}$. Additionally, these channel proteins act as scaffold proteins or signalling hubs via their cytoplasmic domains ${ }^{4,21}$, regulating different key signalling pathways independently of their channel activity ${ }^{4,22-24}$. Several Cx proteins are expressed in developing and mature skeletal tissues ${ }^{19,25,26}$. However, Cx43 is the major Cx protein expressed in chondrocytes, synovial cells and bone cells ${ }^{10,12,27-29}$ and it has been involved in normal development and function of joint tissues ${ }^{25,30-32}$, and in joint disorders including age-related bone $\operatorname{loss}^{33}$, rheumatoid arthritis ${ }^{16,34}$ and $\mathrm{OA}$ progression $^{10,12,25,27-29}$. 
During tissue regeneration and following injury, the dedifferentiation, redifferentiation and senescence processes play finely tuned temporal and spatial roles to reverse the loss of tissue in a precise way ${ }^{35,36}$. Cx43, via channel-dependent and -independent functions, has been involved in different phases of tissue regeneration including in controlling acute and chronic inflammation, cell differentiation, migration, proliferation or cellular reprogramming and lately in cellular senescence $22-24,37,38$. Results from our lab and others, demonstrated that $\mathrm{Cx} 43$ is a target of interest for the treatment of OA in order to stop cartilage degradation and to restore regeneration. Overexpression of Cx43 and enhancement of the GJIC in osteoarthritic chondrocytes (OACs) compromise the ability of these cells to re-differentiate, promoting a stem-like state by activating the chondrocyte-mesenchymal transition factor Twist-1 and p53/p21mediated cellular senescence ${ }^{4}$. However we have recently demonstrated that this is a reversible loss, because downregulation of $\mathrm{Cx} 43$ reduces stemness, hypertrophy markers, senescence and therefore MMPs and proinflammatory mediators, and improves Col2A and proteoglycans' levels in monolayer and 3D cultures ${ }^{4}$. Interestingly, this Cx43-sensitive circuit regulates joint inflammation, cellular plasticity and accumulation of senescent chondrocytes ${ }^{4}$. The downregulation of Cx43 in human osteoarthritic chondrocytes, using CRISPR technology and the GJ inhibitor carbenoxolone, restores chondrocyte redifferentiation and decreases the propensity of chondrocytes to undergo cellular senescence by downregulating Twist-1 and p53/p21/p16 and inhibiting the nuclear translocation of the master regulator of senescence secretory phenotype (SASP) NF-kB ${ }^{4}$. These results clearly indicate that Cx43 controls senescence and acts as a molecular switch in chondrocyte phenotypes within a wound healing process ${ }^{4}$. In fact, downregulation of Cx43 in different wound healing disorders halts disease progression by restoring tissue regeneration ${ }^{39-43}$.

In this study we describe the use of small molecules, based on olive phenolic compounds, to downregulate $\mathrm{Cx} 43$ in $\mathrm{OA}$ using 2D and human 3D cartilage models. We have found that oleuropein decreases Cx43 promoter activity and GJIC, thus enhancing osteogenesis and chondrogenesis in hMSCs and redifferentiation of OACs. Besides, downregulation of Cx43 by olive-derived small polyphenols in OACs reduces cellular senescence in chondrocytes, bone and synovial cells and the inflammatory and catabolic activity related with cartilage degradation in OA. Thus, 
these molecules by targeting $\mathrm{Cx} 43$ and senescence may be attractive candidates for tissue engineering and regenerative medicine strategies for $\mathrm{OA}$ treatment and for promotion of proper cartilage and joint repair in these patients.

\section{Results.}

Olive-derived polyphenols including oleuropein impair adipogenesis and enhance the chondrogenic and osteogenic ability of hMSCs. Cx43, altered during $\mathrm{OA}^{27}$, is also a critical regulator of hMSCs differentiation ${ }^{44-46}$. Based on previous reported results from our group ${ }^{4,27,30,47,48}$, we used a small-scale screening to identify compounds that downregulate Cx43 (data not shown), and we have identified compounds such as the small molecule oleuropein (Fig. 1a). Oleuropein and an olive-extract (OE) containing $40 \%$ of oleuropein significantly reduced Cx43 protein levels in OACs treated with different concentrations for 2 hours, as detected by western-blot and by flow cytometry (Fig 1a). Importantly, polyphenols comprising oleuropein have been previously proposed and patented for the treatment of OA due to their ability to stimulate cartilage anabolism and prevent inflammation ${ }^{49-51}$. An MTT assay showed no effect of $0.1,1$ or $10 \mu \mathrm{M}$ oleuropein on the cell viability (O.D. 570 $\mathrm{nm}$ ) of primary chondrocytes and hMSCs (Fig. 1b). We next examined hMSCs differentiation capacity in the presence of oleuropein (Fig. 1c, 1d and Supplementary Fig. 1). In accordance with previous results ${ }^{52}$, oleuropein-treated hMSCs showed significantly less in adipogenic differentiation detected by oil red $\mathrm{O}$ for lipid staining and by peroxisome proliferator-activated receptor (PPARY) gene expression, whereas osteogenesis, examined by alizarin red staining of calcium deposits and by osteocalcin (OSTCN) gene expression, was significantly increased (Fig. 1c and Supplementary Fig. 1a and 1b). Furthermore, a 3D micromass culture system using chondrogenic medium supplemented with $10 \mu \mathrm{M}$ oleuropein or $\mathrm{OE}$ revealed an increase in ECM properties, reflecting a greater degree of chondrogenic differentiation within the 3D structure, with higher levels of Col2A1 deposited in the ECM and increased levels of aggrecan (ACAN) gene expression (Fig. 1d and Supplementary Fig. 1C). Remarkably decreased Cx43 gene expression was detected in hMSCs under the three differentiation conditions (adipogenesis, osteogenesis and chondrogenesis), this effect being more pronounced during chondrogenesis (Supplementary Fig 2a) and in concordance with a previous report ${ }^{53}$ (Fig. 1e and 1f). In this study, we have also observed changes in the Cx43 phosphorylation patterns at 7 and 14 days of chondrogenic differentiation by western blot (Fig. 1e), which can 
affect Cx43 stability and channel activity ${ }^{54}$. Different Cx43 phosphorylation patterns were also detected during adipogenesis and osteogenesis, suggesting differential regulation of $\mathrm{Cx} 43$ during hMSCs differentiation (Supplementary Fig. 2b). The treatment of hMSCs during chondrogenic differentiation with $10 \mu \mathrm{M}$ oleuropein for 14 days caused an additional 1.7 fold reduction in Cx43 gene expression (Fig. 1f). In concordance with these results, OACs' differentiation (redifferentiation), for 7 days with chondrogenic medium supplemented with $10 \mu \mathrm{M}$ oleuropein, led to a significant decrease in the $\mathrm{Cx} 43$ protein levels without impact on $\mathrm{Cx} 43$ phosphorylation patterns (Fig. 1g).

It is important to note that these results were obtained during differentiation of hMSCs under osteogenesis and chondrogenesis (Fig. 1c-f) and dedifferentiated chondrocytes from patients (OACs) in normal and in chondrogenic medium (Fig. 1a and Fig. 1g). However, the treatment of undifferentiated hMSCs cultured in basal growth medium with oleuropein or OE for 2 hours increased Cx43 levels (Supplementary Fig. 3a and 3b) and GJIC (Supplementary Fig. 3c), indicating that the effect of these olive derived polyphenols may be different depending on the cellular context.

\section{Downregulation of $\mathrm{Cx43}$ activity by oleuropein downregulates the EMT} transcription factor Twist-1 and enhances redifferentiation of OACs. Because oleuropein and the OE modulate $\mathrm{Cx} 43$ in OACs and the differentiation capacity of hMSCs, we expected that these compounds might modify de- and redifferentiation of OACs. Oleuropein treatment of primary chondrocytes isolated from patients with OA (OACs) decreased the Cx43 protein levels, as detected by western blot and flow cytometry (Fig. 1a and Fig 1g). We have previously found that intercellular coupling via GJ channels participates in the effects of Cx43 on the OAC phenotype ${ }^{4}$. Interestingly, oleuropein modulation of Cx43 significantly reduced GJIC in OACs, as detected by scrape loading/dye uptake assay and flow cytometry analysis (Fig. 2a), but not in healthy chondrocytes, with dye transfer through GJ channels being more reduced in OACs than in healthy chondrocytes (Fig. 2b). The decrease in Cx43 GJIC and protein levels were correlated with a significant reduction in the levels of the stemness markers CD105 and CD166 after 7 days in culture in presence of $10 \mu \mathrm{M}$ oleuropein as detected by western-blot and flow cytometry (Fig. 2c). This result was consistent with our previous observations, where CD105 and CD166 stemness 
markers were reduced when $\mathrm{Cx} 43$ was downregulated or when OACs were grown in chondrogenic medium to enhance redifferentiation for 14 days ${ }^{4}$. In fact, oleuropein effectively improved the phenotype of chondrocytes isolated from human $\mathrm{OA}$ cartilage grown in culture as a monolayer detected by Col2A1 levels and synthesis of proinflammatory mediators and MMP-3 (Fig. $2 \mathrm{~d}$ and 2e). Treatment with $10 \mu \mathrm{M}$ oleuropein for $2 \mathrm{~h}$ was enough to reduce overall Cx43 positivity and to increase Col2A1 protein levels (Fig. 2d) and decrease interleukin 6 (IL-6), COX-2, IL-1B and MMP-3 gene expression (Fig. 2e).

Next, we sought to confirm whether oleuropein would target chondrocyte plasticity in 3D cultures. OACs were grown as a micromass pellet culture in chondrogenic medium supplemented with different concentrations of oleuropein (Fig. 2f). The enhancement of fully differentiated chondrocytes in chondrogenic medium improved the micromass matrix structure of OACs in 3D culture (Fig. 2f). Oleuropein and OE further decreased $\mathrm{Cx} 43$ levels (Fig. 2g) and further increased the deposition of proteoglycans detected by toluidine blue staining and Col2A1 detected by immunohistochemistry (Fig. 2f).

To further explore the effect of oleuropein on cell plasticity, OACs were grown in adipogenic medium in presence of $10 \mu \mathrm{M}$ oleuropein. Notably, oleuropein significantly decreased the adipogenic differentiation of OACs (Fig. 2h). However, oleuropein treatment increased the osteogenic differentiation of OACs when these cells were grown in osteogenic medium with $10 \mu \mathrm{M}$ oleuropein for 14 days (Supplementary Fig. 4). Consistent with this finding, previous studies have suggested a direct transformation of mature chondrocytes into osteoblasts and osteocytes ${ }^{55-57}$, reemphasizing the effect of oleuropein as a modulator of cell plasticity in chondrocytes, enhancing OACs redifferentiation to mature chondrocytes (and transdifferentiation into bone cells).

Upregulation of $\mathrm{Cx} 43$ in $\mathrm{OA}$ involves dedifferentiation via chondrocyte-tomesenchymal transition (CMT) by Twist-1 activation, which was also reported in OA cartilage $^{4,58,59}$. After oleuropein treatment, OACs showed a significant reduction in the nuclear localization of Twist-1 detected by western-blot of nuclear extracts (Fig. 2i). Furthermore, immunofluorescence assays showed that the nuclear localization of Twist-1 transcription factor in the presence of an arthritic insult (oligomycin), was 
attenuated when $\mathrm{Cx} 43$ protein levels were reduced by oleuropein treatment of OACs in monolayer culture (Fig. 2j). In addition to Twist-1, oleuropein treatment in OACs led to a significant reduction of the expression of mesenchymal and EMT markers Ncadherin and vimentin (Fig. 2k).

Oleuropein modulates Cx43 gene promoter activity. Mechanisms that underlie the effects of oleuropein or the OE containing oleuropein and other small molecular polyphenols may be multifactorial. Yet, in chondrocytes decreased Cx43 protein levels were detected when OACs and the human chondrocyte cell line T/C-28a2 were treated with $10 \mu \mathrm{M}$ oleuropein (Fig. 1a and 3a). However, changes in Cx43 protein levels were not evident when T/C-28a2 transfected with a vector to overexpress $\mathrm{C} x 43$ was treated with $10 \mu \mathrm{M}$ oleuropein (Fig. 3a, bottom), suggesting that oleuropein in chondrocytes may affect $\mathrm{Cx} 43$ gene promoter activity rather than protein stability. We thus measured whether oleuropein affected the activity of the Cx43 gene promoter using a real-time reporter system. Human T/C-28a2 chondrocytes were transfected with a firefly luciferase reporter vector containing the regulatory regions of the $\mathrm{C} \times 43$ promoter and incubated for $1 \mathrm{~h}$ with $10 \mu \mathrm{M}$ oleuropein and $5 \mu \mathrm{g} / \mathrm{ml}$ of the mitochondrial inhibitor oligomycin, which inhibits ATP synthase by blocking its proton channel $\mathrm{l}^{60,61}$ and enhances Cx43 gene expression (Fig. 2j, Fig 3b). In fact, this small molecule was previously used for in vitro and in vivo models of arthritis to induce inflammation and cartilage degradation ${ }^{62}$. Cx43 promoter activity (detected by luminescence) decreased after oleuropein treatment (Fig. 3b). Oligomycin increased the luminescence signals, and the effect of oligomycin was significantly attenuated in the presence of oleuropein (Fig. 3b), which was correlated with the Cx43 mRNA levels detected by RT-qPCR (Fig. 3b). The luminescence signals strongly correlated with the effects of oleuropein and oligomycin on protein levels detected by immunofluorescence (Fig. 3c) and western blot (Fig. 2j) when OACs were grown in monolayer culture. These results indicate that oleuropein affects $\mathrm{Cx} 43$ (gene, GJA1) promoter activity, thus downregulating Cx43 protein levels (Fig. 1a and Fig. 3a-c) and GJIC in OACs (Fig. 2b).

Oleuropein enhances elimination of senescent cells. Senescent cells through secretion of SASP promote dedifferentiation and reprogramming in neighbouring cells in the context of tissue injury ${ }^{63,64}$. OACs treated with $10 \mu \mathrm{M}$ oleuropein for 7 or 14 days in growth medium showed a significant reduction of senescent cells 
accumulated after 5 days of primary culture (Fig. 4a) and detected by betagalactosidase activity measured by flow cytometry (Fig. 4a). Consistent with these models, Cx43 upregulation due to oligomycin insult (for 24 hours) significantly contributed to increase cellular senescence in OACs accumulated after 5 days in monolayer (Fig. 4b, left), whereas co-treatment with oleuropein significantly halted the accumulation of senescent cells, as measured by the quantitative flow cytometry analysis of beta-galactosidase activity (24 hours under treatments) (Fig. 4b, left) and beta-galactosidase staining ( 7 days under treatments) (Fig. 4b, right). Interestingly, increased levels of Cx43 were detected when the chondrocyte cell line T/C-28a2 was treated with $50 \mu \mathrm{g} / \mathrm{mL}$ of bleomycin for $24 \mathrm{~h}$ hours and the exposure to oleuropein for 24 hours, after bleomycin treatment, reduced both the number of senescent cells detected by B-galactosidase activity and Cx43 protein levels detected by western-blot (Supplementary Fig. 5). In concordance with these results, the treatment of OACs with $10 \mu \mathrm{M}$ oleuropein for 2 hours led to a decrease in the levels of the senescence biomarker p16 ${ }^{\text {INK4a }}$ (Fig. 4c) and p53 and p21 protein levels (Fig. 4d). Cellular senescence and the senescence-associated secretory phenotype (SASP) convert senescent cells into proinflammatory cells by secreting factors such as IL-6 ${ }^{65,66}$. Oleuropein reduced the accumulation of senescent cells and attenuated oligomycininduced SASP secretion detected by IL-6 and COX-2 gene expression in chondrocytes (Fig. 4e). This effect was also detected to a lesser extent for IL-1B. (Fig. 4e). The secretory activity of senescent cells (SASP), including IL-6 gene expression can be activated by the master regulator of SASP, NF- $\mathrm{KB}^{67,68}$. Immunofluorescence assays showed that NF-kB (p65) activation detected by nuclear translocation in OACs by TNFa was diminished when cells were exposed to $10 \mu \mathrm{M}$ oleuropein for $1 \mathrm{~h}$ (Fig. 4f). Oleuropein also protected from the increase of Cx43 under TNFa treatment (Fig. 4f, left). Further, NF-kB nuclear translocation was partially abolished in OACs after 5 days in primary culture and treated with $10 \mu \mathrm{M}$ oleuropein for only 2 hours and detected by western-blot (nuclear extracts) (Fig. 4g).

To further test the senolytic activity of oleuropein, synovial and bone cells isolated from synovium and spongy bone from joints from OA patients were treated with 10 $\mu \mathrm{M}$ oleuropein (Fig. 4h -0). We observed decreased Cx43 protein levels after only 2 hours under oleuropein exposure (Fig. $4 \mathrm{~h}$ and $4 \mathrm{l}$ ) together with a significant reduction in senescent cells accumulated after 5 days in primary culture and detected by Bgalactosidase activity (Fig. $4 \mathrm{i}$ and $4 \mathrm{~m}$ ) and confirmed by RT-qPCR to test the 
expression of the senescence biomarker p16 ${ }^{\mathrm{INK} 4 \mathrm{a}}$ (Fig. $4 \mathrm{j}$ and $4 \mathrm{n}$ ) and the synthesis of the SASP factors IL1-B, COX-2 and IL-6 (Fig. 4k and 40).

\section{Discussion.}

Chondrocyte phenotypic changes are common response to insults and during $O A$ progression ${ }^{4}$. Previous data reported by our group demonstrate that downregulation of Cx43 improves cell phenotype protecting chondrocytes from dedifferentiation (via EMT transcription factors such as Twist-1) and senescence (via p53 activation) ${ }^{4}$. Although oleuropein was reported to protect from OA progression ${ }^{69-73}$, there was no solid evidence about the underlying molecular mechanisms involved in this effect. Our results are consistent with previous studies showing the protective effect of oleuropein and olive derived polyphenols in $O A^{69,71}$ and that $\mathrm{Cx} 43$ downregulation have significant effects on chondrocyte phenotype $e^{4,16,30}$. In our study, we show that oleuropein modulates the activity of the $\mathrm{Cx} 43$ gene promoter, reducing $\mathrm{C} \times 43$ and GJIC in OACs and hMSCs. Indeed, our data indicate that downregulation of Cx43 by oleuropein in OACs in 3D and monolayer culture improves cell phenotype by protecting chondrocytes from the EMT transcription factor Twist-1 activation under experimental insults and from accumulation of senescent cells (Fig. 2 and Fig. 4). This is the first study that demonstrated one of the potential mechanisms of oleuropein in OACs, synovial and bone cells (Fig 4) from patients and in hMSCs (Fig. 1), with important applications in regenerative medicine.

Experimental, clinical and epidemiological data have pointed out the beneficial effects of polyphenols in wound healing in multiple ageing-related disorders ${ }^{74-77}$, making these compounds effective candidates in the context of preventive nutrition and for the development of new therapeutic strategies ${ }^{77-81}$. Oleuropein is the most abundant polyphenol in the leaves and fruit of the olive plant (e.g. Olea europaea L.) and is a potent antioxidant agent with anti-tumour and anti-inflammatory properties ${ }^{82,83}$. The mechanism of action this polyphenol is under study ${ }^{82}$, and show that oleuropein and its major metabolite hydroxytyrosol have antioxidant activity by inhibition and/or scavenging of $\mathrm{ROS}^{84,85}$, which has been suggested to reduce NF-kB activation $^{86-89}$. Other mechanistic studies implicate nitric oxide (NO) production ${ }^{89-92}$ or autophagy and inhibition of the mammalian target of rapamycin (mTOR) ${ }^{93-95}$. Here, we show that oleuropein directly regulates Cx43 gene promoter activity leading to downregulation of GJIC (Fig. 2a and Fig. 3b). Interestingly, in hMSCs oleuropein 
reduces Cx43 and GJIC only under differentiation processes and increases Cx43 in undifferentiated hMSCs (Fig 1e, 1f, Supplementary Fig. 2 and Supplementary Fig. 3). These results indicate that the activity of this molecule in the Cx43 gene promoter depends on the context. In fact, a gene expression profiling study has suggested that oleuropein affects the expression of multiple genes involved in oxidative stress, inflammation, fibrosis, cell proliferation and differentiation among others ${ }^{96}$, which implies that the mechanisms that underlie the beneficial effects of this molecule may be multifactorial and depend on the conditions ${ }^{96}$. These observations emphasize the importance of Cx43-mediated effects of oleuropein specifically during the differentiation process. Also, our results show that the effects of oleuropein are often equal or even smaller than those of olive extract that contains $41.5 \%$ of oleuropein at equivalent doses. This observation suggests that other polyphenols or compounds, such as the hydroxytyrosol, may synergize with oleuropein activity in chondrocytes $^{97,98}$. On the other hand, the treatment of hMSCs with oleuropein or OE, in chondrogenic, osteogenic or adipogenic differentiation medium leads to downregulation of Cx43 and GJIC, enhancing osteogenesis and chondrogenesis, but reducing adipogenesis. This differential sensitivity of hMSCs to oleuropein and OE may have potential applications in preventive and regenerative medicine in other bone and cartilage disorders e.g. by reducing ectopic adipocyte accumulation in bone marrow cavities and improving osteogenesis during bone repair ${ }^{99-101}$.

Our data show decreased Cx43 levels with decreased GJ activity in the presence of oleuropein in OACs and in differentiating hMSCs, but increased Cx43 levels with increased GJIC in undifferentiated hMSCs, suggesting that the effect of this molecule on GJIC depends on its effect on Cx43 levels (or subcellular localization). In fact, phosphorylation of Cx43 affects protein stability and GJIC activity ${ }^{54,102,103}$ and we have detected changes in Cx43 phosphorylation pattern during hMSCs differentiation (chondrogenesis, osteogenesis and adipogenesis) but not under oleuropein treatment (in hMSCs and OACs) indicating that oleuropein affects Cx43 levels more than protein or gap junction plaque stability or regulation of the activity of these intercellular channels. Further, Cx43 channel-independent activities involve its signalling hub's ability to recruit proteins to the membrane $e^{24,104-106}$ or its ability to control gene transcription by nuclear translocation and binding to RNA polymerase II and gene promoters ${ }^{107}$. We have recently reported that overactivity of Cx43 in OACs compromise the ability of these cells to redifferentiate by maintaining the stem-like 
state enhanced by Twist-1 activity and tissue remodelling and proinflammatory agents such as IL-1 $\beta^{4}$. Here, we show that oleuropein restores chondrocyte phenotype detected by reduced levels of the stem-markers CD105, CD166, N-cad and vimentin (Fig. 2c, 2k). The effect of oleuropein in chondrocyte plasticity correlated with activation of redifferentiation via downregulation of $\mathrm{Cx} 43$ and Twist-1 (Fig.2i, 2j, 2k), leading to increased levels of proteoglycans and Col2A1 together with a decrease in the levels of inflammatory cytokines and metalloproteinases such as IL-1B and MMP-3 (Fig. 2d, 2e, 2f).

In cell culture and in osteoarthritic cartilage, OACs undergo dedifferentiation and senescence ${ }^{4,108,109}$. Elimination of senescent cells in vivo using the senolytic drug UBX0101 has been demonstrated to improve cartilage regeneration after articular joint injury in mice ${ }^{3}$. Here we show that oleuropein reduces cellular senescence in OACs, synovial and bone cells in monolayer and protects from accumulation of senescent cells under an arthritic insult (Fig. 4a, 4b, 4i and $4 \mathrm{~m}$ ). NF-kB has been shown to cooperatively regulate the inflammatory components of the SASP, together with other factors ${ }^{68,110-112}$. In this study the reduction of senescence is accompanied by reduced activity of the NF-kB transcription factor and therefore reduced synthesis of SASP including those encoding IL-6, IL-1B and COX-2 (Fig. 4e). Notably, these components enhance inflammation, senescence and activate dedifferentiation and cellular reprogramming of nearby non-senescent cells (e.g. via IL6) ${ }^{63,64}$, contributing to the stem-like state of chondrocytes in $\mathrm{OA}$ and to the accumulation of senescent cells. Accordingly, we have previously reported that upregulation of $\mathrm{Cx} 43$ leads to p53/p16 upregulation and senescence ${ }^{4}$. Using the T/C-28a2 human chondrocyte cell line with a Cx43 overexpression vector and a CRISPR/Cas9-mediated heterozygous Cx43 gene knockout cell line we have demonstrated that $\mathrm{Cx} 43$ is an upstream effector of both senescence (involving p53 and p16 pathways) and NF-kB activation (SASP synthesis) ${ }^{4}$. However IL-1 signalling was also shown to be an upstream effector of NF-kB ${ }^{113-115}$. IL-1 gene expression is activated by $\mathrm{C} \times 43^{4,29}$ and vice versa, providing another level of positive feedback loop involved in cartilage degradation in OA.

Cellular reprogramming, dedifferentiation via EMT and senescence play active roles during tissue regeneration ${ }^{116}$. These processes are activated in response to damage and involve dedifferentiation, reprogramming, inflammation and ECM remodelling 
and resolve with redifferentiation and elimination of senescent cells ${ }^{116}$. Accumulation of dedifferentiated (stem-like cells) and senescent cells leads to impaired tissue regeneration and fibrosis with loss of tissue function ${ }^{117,118}$ (Fig. 5). Understanding and manipulating the complex $\mathrm{Cx} 43$ signalling appears to be very challenging but would expand our opportunities for modulating wound-healing related disorders such as OA. So far, our results indicate that molecules that reduce $\mathrm{Cx} 43$ levels in OA such as oleuropein will potentially restore cartilage repair in OA by activating chondrocyte redifferentiation and by eliminating senescent cells, contributing to creating a regeneration-permissive environment in OA patients.

Consistent with previous studies, oleuropein shows a potent senolytic and antiinflammatory effect by reducing IL-1B, IL- 6 and COX-2 gene expression and NF-kB activation $^{119}$. Our study is the first to demonstrate the effect of this polyphenol on Cx43 activity and senescence. Our results show that oleuropein downregulates Cx43 in OACs, enhancing chondrocyte redifferentiation, together with a decrease in cellular senescence and SASP activated by NF-kB (Fig. 5). Besides, modulation of Cx43 by oleuropein shifts the hMSCs differentiation capacity towards osteogenic and chondrogenic lineages, while decreasing adipogenic differentiation. These effects may be useful in cell therapy approaches that aim to promote cartilage and bone regeneration because the osteogenesis/adipogenesis switch has been associated with different bone disorders characterized by reduced bone formation and increased bone marrow fat accumulation ${ }^{99,100}$. Altogether, these findings indicate that oleuropein controls $\mathrm{Cx} 43$ gene expression and acts as a potent senolytic drug that may serve as a potential agent to improve both the effectiveness of stem cell therapy and cartilage and joint regeneration in patients to stop OA progression, by restoring tissue regeneration.

\section{Experimental procedures.}

Tissue collection, processing and cell culture. Collection and processing of cartilage from human knees and femoral heads from adult donors undergoing joint surgery were performed as previously described ${ }^{28}$. The study was conducted with the approval of the institutional ethics committee (C.0003333, 2012/094 \& 2015/029) after the acquisition of the signed informed consents. Samples from healthy, moderate OA (grades I-II) and high OA (grades III-IV) groups were selected for this study, which were classified according to their medical record data and histological 
analysis, as previously described ${ }^{27} .25$ human samples were used for this study. Each experiment was conducted with at least three different patients/samples. Primary chondrocytes, isolated from fresh cartilage as previously described ${ }^{28}$, were cultured in Dulbecco's Modified Eagle's medium (DMEM) supplemented with 100 $\mathrm{U} / \mathrm{ml}$ penicillin, $100 \mu \mathrm{g} / \mathrm{ml}$ streptomycin and $10 \%$ foetal bovine serum (FBS), all from Gibco, Thermo Fisher Scientific. The chondrocyte cell line T/C-28a2, kindly donated by Dr. Goldring from the Hospital for Special Surgery (New York, USA), was cultured in DMEM supplemented with $10 \%$ FBS, $100 \mathrm{U} / \mathrm{ml}$ penicillin and $100 \mu \mathrm{g} / \mathrm{ml}$ streptomycin. Human mesenchymal stem cells were obtained with signed informed consent from bone marrow donors (Hospital Universitario Reina Sofía, Córdoba, Spain) and from subcutaneous inguinal fat from healthy individuals (Hospital Universitario Ramón y Cajal, Madrid, Spain). hMSCs were cultured in a-minimum essential medium (a-MEM; Lonza) supplemented with 10\% FBS, $100 \mathrm{U} / \mathrm{ml}$ penicillin, $100 \mu \mathrm{g} / \mathrm{ml}$ streptomycin, $2 \mathrm{mM}$ GlutaMax (Gibco, Thermo Fisher Scientific) and 1 $\mathrm{ng} / \mathrm{ml}$ recombinant human fibroblast growth factor-2 (rhFGF-2; Immunotools) or in MesenPRO RS ${ }^{\mathrm{TM}}$ Medium supplemented with $100 \mathrm{U} / \mathrm{ml}$ penicillin and $100 \mu \mathrm{g} / \mathrm{ml}$ streptomycin.

For cell treatments, oleuropein with $\geq 90 \%$ purity was purchased from Extrasynthese (0204) (Lyon, France) and OE was donated by the Clinical Management Unit of Endocrinology and Nutrition (IMIBIC, Córdoba, Spain). This extract contains $41.5 \%$ oleuropein and was dissolved in culture medium to a stock concentration of $13 \mathrm{mg} / \mathrm{ml}$ (equivalent to $100 \mu \mathrm{M}$ oleuropein). Both compounds were dissolved in the cell culture medium and added to the cells for short-term (1-2 h) or long-term (7-21 days) treatments. Cell insults were performed with either $5 \mu \mathrm{g} / \mathrm{ml}$ oligomycin (SigmaAldrich, O4876) or $10 \mathrm{ng} / \mathrm{mL}$ TNFa (Immunotools, 11343013), for $1 \mathrm{~h}$.

Cell viability assay. Cells in 96-well culture plates were treated with $0.1 \mu \mathrm{M}, 1 \mu \mathrm{M}$, $10 \mu \mathrm{M}$ and $10 \mathrm{mM}$ oleuropein for $17 \mathrm{~h}$. Drug cytotoxicity was evaluated by the colorimetric MTT (3-(4,5-dimethylthiazol-2-yl)-2,5-diphenyltetrazolium bromide) assay (Cell Proliferation Kit I, Roche) with a NanoQuant microplate reader (Tecan Trading AG, Switzerland) at $570 \mathrm{~nm}$.

Adipogenic differentiation. Adipogenic differentiation was performed as previously described ${ }^{4}$. hMSCs or chondrocytes at a $80-100 \%$ confluence were incubated with adipogenic medium (hMSC Adipogenic Differentiation Bullekit ${ }^{\mathrm{TM}}$, Lonza) for 21 days. 
The medium was changed every 2 - 3 days, with the addition of $0.1,1$ or $10 \mu \mathrm{M}$ oleuropein or OE when necessary. Adipogenic differentiation was evaluated by the RNA expression of PPARY (5'-GCGATTCCTTC ACTGATACACTG-3', 5'-GAGTGGGAGTGGTCTTCCATTAC-3') and by oil red O staining with a freshly prepared $60 \%(\mathrm{v} / \mathrm{v})$ oil red $\mathrm{O}$ solution. Lipid droplet-containing cells were analysed with the ImageJ software (version 1.48) and adipogenic differentiation was evaluated as the ratio of positive droplet-containing cells to the total number of cells.

Osteogenic differentiation. For osteogenic differentiation, cells were differentiated for 21 days with osteogenic medium (StemPro® Osteogenesis Differentiation Kit, Gibco, Thermo Fisher Scientific), changing the medium every 2 - 3 days with the addition of $0.1,1$ or $10 \mu \mathrm{M}$ oleuropein or OE. Osteogenesis was evaluated by the RNA expression of OSTCN (5'-CCATGAGAGCCCTCACACTCC-3', 5'GGTCAGCCAACTCGTCACAGTC-3') and by alizarin red $S$ staining with a $2 \%(w / v)$ alizarin red $S$ solution (Sigma-Aldrich). Slides were imaged in an Olympus BX61 microscope, and red positivity was analysed using ImageJ software (version 1.48).

Chondrogenic differentiation. hMSCs or chondrocytes were differentiated with chondrogenic medium (StemPro® Chondrogenesis Differentiation Kit, Gibco, Thermo Fisher Scientific) as micromasses for 30 days in the presence of chondrogenic medium, with or without oleuropein or OE addition. Micromasses were embedded in Tissue-Tek ${ }^{\circledR} \mathrm{OCT}^{\mathrm{TM}}$ (Sakura) and chondrogenesis was evaluated with ECM-specific stains (toluidine blue, safranin O-fast green) and by ACAN RNA expression (5'CAGAACAACTCGGGGAACAT-3', 5'-GCACAATTGGAACCCTGACT-3').

Scrape loading/dye transfer (SL/DT) assay. In order to evaluate GJIC, a SL/DT assay was performed as previously described ${ }^{4,28}$. Confluent cells were cultured for 16 $\mathrm{h}$ of in DMEM without FBS supplementation, and then oleuropein or olive leaf extract $(\mathrm{OE})$ was added to the medium for $2 \mathrm{~h}$. Next, a $0.4 \%(\mathrm{w} / \mathrm{v})$ solution of lucifer yellow (LY) (Cell Projects Ltd(C, Kent, UK) in PBS was loaded and two distant scrapes were made across the culture plate. The LY solution was absorbed by the damaged cells, which were allowed to transfer the dye for $3 \mathrm{~min}$ at $37^{\circ} \mathrm{C}$. LY transfer was evaluated in a Nikon Eclipse Ti fluorescent microscope, and the number of dye-positive cells 
(LY transfer) from the cut site to the farthest detectable uptake of LY reflects the GJ connectivity between cells. The score was calculated as previously reported ${ }^{120,121}$.

GJ connectivity by flow cytometry. Equal numbers of cells were incubated for $1 \mathrm{~h}$ at $37^{\circ} \mathrm{C}$ with either $1 \mu \mathrm{M}$ of the membrane dye Dil (Invitrogen, Thermo Fisher Scientific) or $1 \mu \mathrm{M}$ calcein-AM (Invitrogen, Thermo Fisher Scientific), a cellpermeable dye that is retained in the cytosol and can be transferred by GJs.. Then, cells were washed 3 times with PBS and co-cultured for $2 \mathrm{~h}$ in a 1:2 ratio (calceindonors:Dil-recipient cells). The percentage of $\mathrm{Dil}^{+} \mathrm{Calc}^{+}$cells was analysed by flow cytometry and compared with that of unlabelled cells and to a 1:1 mixture of labelled cells mixed immediately before the analysis (Time $=0 \mathrm{~h}$ ).

Western blot. For protein analysis, cells were lysed in ice-cold lysis buffer (150 mM $\mathrm{NaCl}, 50 \mathrm{mM}$ Tris-HCl pH 7.5, 5 mM EDTA pH 8, 0.5\% v/v Nonidet P-40, 0.1\% (w/v) SDS, $0.5 \%(\mathrm{v} / \mathrm{v})$ sarkosyl) supplemented with $5 \mu \mathrm{g} / \mathrm{ml}$ protease inhibitor cocktail and $1 \mathrm{mM}$ phenylmethylsulfonyl fluoride (PMSF; Sigma-Aldrich). Nuclear protein isolation was performed with the NE-PER ${ }^{\mathrm{TM}}$ kit (Thermo Fisher Scientific), according to the manufacturer's instructions. Proteins were separated on a 10\% SDS-PAGE and transferred to a polyvinylidene fluoride (PVDF) membrane (Millipore Co., Bedford, MA). Primary antibody incubation was performed overnight at $4^{\circ} \mathrm{C}$, and HRPsecondary antibody incubation was performed at RT for $1 \mathrm{~h}$. Signal developing was performed with the Pierce ${ }^{T M}$ ECL Western Blotting Substrate in either a LAS-300 Imager (Fujifilm) or an Amersham Imager 600 (GE Healthcare). The following primary antibodies were used: a-tubulin (Sigma-Aldrich, T9026), Cx43 (SigmaAldrich, C6219), Twist-1 (Santa Cruz Biotechnology, sc-81417), CD166 (Santa Cruz Biotechnology, sc-74558), p16 ${ }^{\text {INK4a }}$ (Abcam, ab108349), p21 (Santa Cruz Biotechnology, sc-6246), p53 (Santa Cruz Biotechnology, sc-126), NF-kB (Santa Cruz Biotechnology, sc-8008) and lamin A (Santa Cruz Biotechnology, sc-20680).

Antigen expression analysis by flow cytometry. Paraformaldehyde-fixed cells were incubated with phycoerythrin (PE)-conjugated anti-human CD105 (Immunostep, 105PE-100T) or allophycocyanin (APC)-conjugated anti-human CD166 (Immunostep, 1399990314), for $30 \mathrm{~min}$ at $4^{\circ} \mathrm{C}$ in the dark, as previously reported ${ }^{4}$ (Supplementary table 1). Intracellular $\mathrm{Cx} 43$ protein was detected in paraformaldehyde-fixed cells, which were permeabilized with methanol for $30 \mathrm{~min}$ at 
$4^{\circ} \mathrm{C}$. Finally, cells were incubated with APC-conjugated anti-human $\mathrm{Cx} 43$ antibody (R\&D Systems, FAB7737A; Supplementary table 1) for 30 min at $4^{\circ} \mathrm{C}$ in the dark.

Flow cytometry analysis. Between $10.000-20.000$ events were collected on a BD FACSCalibur $^{\mathrm{TM}}$ (Becton Dickinson) flow cytometer with the CellQuest ${ }^{\mathrm{TM}}$ Pro software. Cell debris was discriminated by the forward scatter (FSC) and side scatter (SSC) properties of the cells. Data were analysed with FCS Express 6 Flow software (De Novo Software). The level of positive staining was expressed as the median fluorescence intensity (MFI), with unlabelled cells as negative controls. Gates were placed based on single-labelled controls and by establishing $0.1 \%$ as the cutoff point.

Senescence-associated $\beta$-galactosidase activity. Flow cytometry analysis of $S A \beta G$ activity with the fluorogenic $\beta$-galactosidase substrate fluorescein di- $\beta-D-$ galactopyranoside (FDG; Invitrogen, Thermo Fisher Scientific) was performed as previously described ${ }^{4}$. Briefly, harvested cells were incubated with pre-warmed $2 \mathrm{mM}$ FDG for $3 \mathrm{~min}$ at $37{ }^{\circ} \mathrm{C}$, and fluorescein positivity was analysed on a BD FACSCalibur $^{\top M}$ (Becton Dickinson) flow cytometer. SA $\beta G$ activity was also measured with the Senescence Cells Histochemical Staining Kit (Sigma-Aldrich) according to the manufacturer's protocol.

Immunofluorescence. $2 \%$ paraformaldehyde-fixed cells were incubated for $10 \mathrm{~min}$ with $0.1 \mathrm{M}$ glycine (Sigma-Aldrich). Membrane permeabilization was performed with $0.2 \%$ Triton X-100 (Sigma-Aldrich) in PBS, $10 \mathrm{~min}$ at RT, followed by a 30-min incubation with $1 \%$ BSA and $0.1 \%$ Tween 20 in PBS (PBST). Primary and secondary antibodies were diluted in 1\% BSA in PBST and incubated for $1 \mathrm{~h}$ at RT. Nuclei were stained with $1 \mu \mathrm{g} / \mathrm{ml}$ 4',6-diamidino-2-phenylindole dihydrochloride (DAPI; SigmaAldrich) in the dark for $4 \mathrm{~min}$ at RT. Slides were mounted with glycergel aqueous mounting medium (Dako). The following primary antibodies were used: anti-Cx43 (Sigma-Aldrich, C6129), anti-collagen II (Invitrogen, Thermo Fisher Scientific, MA512789), anti-Twist-1 (sc-81417) and anti-NF-KB (sc-8008) from Santa Cruz Biotechnology. Goat anti-rabbit FITC-conjugated (F-2765, Invitrogen, Thermo Fisher Scientific) and goat anti-mouse Alexa 594-conjugated (A-11032, Invitrogen, Thermo Fisher Scientific) secondary antibodies were used. Fluorescence was analysed by using ImageJ software version 1.48 and is shown as the corrected total cell fluorescence (CTCF). 
Immunohistochemistry. Micromass sections were fixed with acetone for $10 \mathrm{~min}$, PBS-washed and nonspecific endogenous peroxidase activity was quenched with a $3 \%(\mathrm{v} / \mathrm{v})$ hydrogen peroxidase solution (Roche) for $10 \mathrm{~min}$. Primary antibody was incubated for $1 \mathrm{~h}$ at RT, and then slides were washed with PBS and incubated for 10 min with OptiView HQ Universal Linker (Roche) at RT. After three additional PBSwashes, slides were incubated with OptiView HRP Multimer (Roche) for 8 min at RT. Peroxidase activity was developed using a $0.02 \%$ hydrogen peroxidase solution and $0.1 \%$ DAB solution. Sections were then washed in distilled water and, in some cases, counterstained with Gill's hematoxylin (Merck Millipore). Finally, slides were dehydrated with alcohol, cleared with xylene (PanReac AppliChem) and mounted with DePeX (BDH Gun®, VWR).

Immunohistochemistry analysis. For immunohistochemistry analysis quantification, an in-house developed MATLAB program was used. This program first splits RGB images into single channels and applies a 3-level automatic thresholding to the green channel using Otsu's method. Then, it segments the images into 4 discrete classes using the threshold levels. As a final step, the total pixels are obtained from the segmented images belonging to the 3 darker classes by discarding the lightest level in the image (background).

Quantitative PCR. TRIzol ${ }^{\mathrm{TM}}$ reagent (Invitrogen, Thermo Fisher Scientific) was used to isolate total RNA, according to the manufacturer's instructions. Degradation of DNA from samples was ensured by treatment with RNase-free DNase (Invitrogen, Thermo Fisher Scientific). $1 \mu \mathrm{g}$ of total RNA per reaction was used to synthesize cDNA with the SuperScript ${ }^{\circledR}$ VILO ${ }^{\mathrm{TM}}$ cDNA Synthesis Kit (Invitrogen, Thermo Fisher Scientific). Quantitative PCR was performed with the Applied Biosystems ${ }^{\mathrm{TM}}$ PowerUP $^{\mathrm{TM}}$ SYBR $^{\mathrm{TM}}$ Green Master Mix from Applied Biosystems on a real-time PCR instrument (LightCycler ${ }^{\circledR} 480$ System, Roche) using the primers listed in Supplementary Table 2.

Cell transfection. Cx43 was overexpressed, as previously described ${ }^{4}$, in the T/C$28 \mathrm{2} 2$ chondrocyte cell after transfection with a pIRESpuro2 plasmid construct (Clontech) containing the human $\mathrm{Cx} 43$ sequence, kindly provided by Arantxa Tabernero (INCL, University of Salamanca, Spain). Electroporation was performed 
with the Amaxa ${ }^{\circledR}$ Cell Line Nucleofector ${ }^{\circledR}$ Kit $\mathrm{V}$ (Lonza) in a Nucleofector ${ }^{\mathrm{TM}} 2 \mathrm{~b}$ device (Lonza) following the manufacturer's instructions. Transfected cells selection was performed by culturing them with $0.1 \mu \mathrm{g} / \mathrm{ml}$ puromycin dihydrochloride (Tocris).

Luciferase reporter assay. A DNA construct containing the upstream $300 \mathrm{bp}$ regulatory sequence of the human $\mathrm{Cx} 43$ promoter $(-108,+279$, relative to the human Cx43 transcription start site) in a pGL3-Basic vector was kindly donated by Dr. Mustapha Kandouz (Wayne State University, School of Medicine, Detroit, USA). The T/C-28a2 chondrocyte cell line was transfected with $3 \mu \mathrm{g}$ of the plasmid using the Amaxa ${ }^{\circledR}$ Cell Line Nucleofector ${ }^{\circledR}$ Kit $\mathrm{V}$ in a Nucleofector ${ }^{\mathrm{TM}} 2 \mathrm{~b}$ device. After 24 h, cells were treated with $10 \mu \mathrm{M}$ oleuropein and/or $5 \mu \mathrm{g} / \mathrm{ml}$ of the ATP synthase blocker oligomycin (Sigma-Aldrich, O4876) for 1 h. For the luminescence analysis, the Firefly Luciferase Assay Kit from Biotium was used according to the manufacturer's instructions. Briefly, cells were lysed with the Firefly Luciferase Lysis Buffer and the lysate was incubated with a $0.2 \mathrm{mg} / \mathrm{ml}$ luciferin solution. Firefly luminescence was measured in an Infinite 200 PRO Tecan plate reader (Tecan). Luminescence was normalized to the total protein content.

Statistical analysis. Data were analysed using GraphPad Prism software (version 5.00). Unless otherwise indicated, analyses were performed using either the Student's $t$-test or the Mann-Whitney $U$-test. $\mathrm{P}<0.05$ was considered statistically significant.

\section{Acknowledgements.}

This work was supported in part through funding from the Spanish Foundation for Research on Bone and Mineral Metabolism (FEIOMM), grant PRECIPITA-2015000139 from the FECYT-Ministry of Economy and Competitiveness (to M.D.M.), grant PI16/00035 from the Health Institute 'Carlos III' (ISCIII, Spain), the European Regional Development Fund, 'A way of making Europe' from the European Union (to M.D.M.) and a grant from Xunta de Galicia IN607B 2017/21 (to M.D.M.) and predoctoral fellowship to M.V.-E (ED481A-2015/188). We thank members of the CellCOM group for helpful technical suggestions, María Dolores Álvarez Alvariño and Jesús Loureiro for generously collecting tissue samples in the operating room after surgery, Ángel Concha and the Biobank of A Coruña, María Vázquez and Beatriz 
Lema for tissue processing and micromass sectioning. Moisés Blanco for helpful advice for the statistical analysis of experimental data.

\section{Author contributions.}

M.V.-E. designed and performed the experiments, analysed the data and prepared the figures. A.V.-V. assisted with cell culture and chondrocyte isolation from patients' cartilage. C.L.P., M.K., A.C.-D. and A.C.-C. provided drugs and hMSCs from healthy donors. V.M. analysed data. E.F. and J.R.C. contributed clinical and technical advice. J.R.C. also provided cartilage tissue from patients and healthy donors. A.B. designed, performed and helped with various aspects of the flow cytometry experiments and analysis. M.D.M. conceived, directed and supervised the study. M.V.-E. and M.D.M. wrote the manuscript with input from all co-authors. All authors reviewed the manuscript.

\section{References.}

1 Neogi, T. \& Zhang, Y. Epidemiology of osteoarthritis. Rheum Dis Clin North Am 39, 1-19, doi:10.1016/j.rdc.2012.10.004 (2013).

2 Lories, R. J. \& Luyten, F. P. The bone-cartilage unit in osteoarthritis. Nat Rev Rheumatol 7, 43-49, doi:10.1038/nrrheum.2010.197 (2011).

3 Jeon, O. H. et al. Local clearance of senescent cells attenuates the development of post-traumatic osteoarthritis and creates a pro-regenerative environment. Nat Med 23, 775-781, doi:10.1038/nm.4324 (2017).

4 Varela-Eirin, M. et al. Targeting of chondrocyte plasticity via connexin43 modulation attenuates cellular senescence and fosters a pro-regenerative environment in osteoarthritis. Cell death \& disease 9, 1166, doi:10.1038/s41419-0181225-2 (2018).

5 Alsalameh, S., Amin, R., Gemba, T. \& Lotz, M. Identification of mesenchymal progenitor cells in normal and osteoarthritic human articular cartilage. Arthritis Rheum 50, 1522-1532, doi:10.1002/art.20269 (2004).

6 Grogan, S. P., Miyaki, S., Asahara, H., D'Lima, D. D. \& Lotz, M. K. Mesenchymal progenitor cell markers in human articular cartilage: normal distribution and changes in osteoarthritis. Arthritis Res Ther 11, R85, doi:10.1186/ar2719 (2009). 7 Goldring, M. B. \& Marcu, K. B. Cartilage homeostasis in health and rheumatic diseases. Arthritis Res Ther 11, 224, doi:10.1186/ar2592 (2009). 
8 Cross, M. et al. The global burden of hip and knee osteoarthritis: estimates from the global burden of disease 2010 study. Ann Rheum Dis 73, 1323-1330, doi:10.1136/annrheumdis-2013-204763 (2014).

9 Varela-Eirin, M. et al. Cartilage regeneration and ageing: Targeting cellular plasticity in osteoarthritis. Ageing research reviews 42, 56-71, doi:10.1016/j.arr.2017.12.006 (2018).

10 Niger, C., Howell, F. D. \& Stains, J. P. Interleukin-1beta increases gap junctional communication among synovial fibroblasts via the extracellular-signalregulated kinase pathway. Biol Cell 102, 37-49, doi:10.1042/BC20090056 (2009).

11 Knight, M. M., McGlashan, S. R., Garcia, M., Jensen, C. G. \& Poole, C. A. Articular chondrocytes express connexin 43 hemichannels and P2 receptors - a putative mechanoreceptor complex involving the primary cilium? $J$ Anat 214, 275283, doi:10.1111/j.1469-7580.2008.01021.x (2009).

12 Marino, A. A. et al. Increased intercellular communication through gap junctions may contribute to progression of osteoarthritis. Clin Orthop Relat Res, 224232 (2004).

13 Delvaeye, T., Vandenabeele, P., Bultynck, G., Leybaert, L. \& Krysko, D. V. Therapeutic Targeting of Connexin Channels: New Views and Challenges. Trends Mol Med 24, 1036-1053, doi:10.1016/j.molmed.2018.10.005 (2018).

14 Montgomery, J., Ghatnekar, G. S., Grek, C. L., Moyer, K. E. \& Gourdie, R. G. Connexin 43-Based Therapeutics for Dermal Wound Healing. Int $J$ Mol Sci 19, doi:10.3390/ijms19061778 (2018).

15 Cogliati, B. et al. Connexin 43 deficiency accelerates skin wound healing and extracellular matrix remodeling in mice. $J$ Dermatol Sci 79, 50-56, doi:10.1016/j.jdermsci.2015.03.019 (2015).

16 Tsuchida, S. et al. Silencing the expression of connexin 43 decreases inflammation and joint destruction in experimental arthritis. J Orthop Res 31, 525530, doi:10.1002/jor.22263 (2013).

17 Casagrande, D., Stains, J. P. \& Murthi, A. M. Identification of shoulder osteoarthritis biomarkers: comparison between shoulders with and without osteoarthritis. Journal of shoulder and elbow surgery 24, 382-390, doi:10.1016/j.jse.2014.11.039 (2015).

18 Kajiwara, Y. et al. GJA1 (connexin43) is a key regulator of Alzheimer's disease pathogenesis. Acta neuropathologica communications 6, 144 , doi:10.1186/s40478-018-0642-x (2018). 
19 Carpintero-Fernandez, P. et al. Intercellular communication via gap junction channels between chondrocytes and bone cells. Biochimica et biophysica acta. Biomembranes 1860, 2499-2505, doi:10.1016/j.bbamem.2018.09.009 (2018).

20 Laird, D. W. \& Lampe, P. D. Therapeutic strategies targeting connexins. Nat Rev Drug Discov, doi:10.1038/nrd.2018.138 (2018).

21 Gago-Fuentes, R. et al. Proteomic Analysis of Connexin 43 Reveals Novel Interactors Related to Osteoarthritis. Mol Cell Proteomics 14, 1831-1845, doi:10.1074/mcp.M115.050211 (2015).

22 Rhett, J. M. et al. Mechanism of action of the anti-inflammatory connexin43 mimetic peptide JM2. Am J Physiol Cell Physiol 313, C314-C326, doi:10.1152/ajpcell.00229.2016 (2017).

23 De Bock, M., Wang, N., Decrock, E., Bultynck, G. \& Leybaert, L. Intracellular Cleavage of the Cx43 C-Terminal Domain by Matrix-Metalloproteases: A Novel Contributor to Inflammation? Mediators of inflammation 2015, 257471, doi:10.1155/2015/257471 (2015).

24 Yang, Y. et al. A connexin43/YAP axis regulates astroglial-mesenchymal transition in hemoglobin induced astrocyte activation. Cell death and differentiation 25, 1870-1884, doi:10.1038/s41418-018-0137-0 (2018).

25 Donahue, H. J., Qu, R. W. \& Genetos, D. C. Joint diseases: from connexins to gap junctions. Nat Rev Rheumatol 14, 42-51, doi:10.1038/nrrheum.2017.204 (2017). 26 Plotkin, L. I. \& Stains, J. P. Connexins and pannexins in the skeleton: gap junctions, hemichannels and more. Cell Mol Life Sci 72, 2853-2867, doi:10.1007/s00018-015-1963-6 (2015).

27 Mayan, M. D. et al. Human articular chondrocytes express multiple gap junction proteins: differential expression of connexins in normal and osteoarthritic cartilage. Am J Pathol 182, 1337-1346, doi:10.1016/j.ajpath.2012.12.018 S0002-9440(13)00028-X [pii] (2013).

28 Mayan, M. D. et al. Articular chondrocyte network mediated by gap junctions: role in metabolic cartilage homeostasis. Ann Rheum Dis, doi:10.1136/annrheumdis2013-204244

annrheumdis-2013-204244 [pii] (2013).

29 Gupta, A. et al. Connexin43 enhances the expression of osteoarthritisassociated genes in synovial fibroblasts in culture. BMC Musculoskelet Disord 15, 425, doi:10.1186/1471-2474-15-425 (2014). 
30 Gago-Fuentes, R. et al. The C-terminal domain of connexin43 modulates cartilage structure via chondrocyte phenotypic changes. Oncotarget 7, 73055-73067, doi:10.18632/oncotarget.12197 (2016).

31 Plotkin, L. I. \& Bellido, T. Beyond gap junctions: Connexin43 and bone cell signaling. Bone 52, 157-166, doi:10.1016/j.bone.2012.09.030 (2013).

32 Kolomytkin, O. V. et al. Gap junctions in human synovial cells and tissue. $J$ Cell Physiol 184, 110-117, doi:10.1002/(SICI)1097-4652(200007)184:1<110::AIDJCP12>3.0.CO;2-8 (2000).

33 Lloyd, S. A., Lewis, G. S., Zhang, Y., Paul, E. M. \& Donahue, H. J. Connexin 43 deficiency attenuates loss of trabecular bone and prevents suppression of cortical bone formation during unloading. $J$ Bone Miner Res 27, 2359-2372, doi:10.1002/jbmr.1687 (2012).

34 Matsuki, T. et al. Expression of Connexin 43 in Synovial Tissue of Patients With Rheumatoid Arthritis. Archives of rheumatology 31, 55-63, doi:10.5606/ArchRheumatol.2016.5597 (2016).

35 Cheng, F. et al. Vimentin coordinates fibroblast proliferation and keratinocyte differentiation in wound healing via TGF-beta-Slug signaling. Proc Natl Acad Sci U S A 113, E4320-4327, doi:10.1073/pnas.1519197113 (2016).

36 Gurtner, G. C., Werner, S., Barrandon, Y. \& Longaker, M. T. Wound repair and regeneration. Nature 453, 314-321, doi:10.1038/nature07039 (2008).

37 Laird, D. W. Syndromic and non-syndromic disease-linked Cx43 mutations. FEBS Lett 588, 1339-1348, doi:10.1016/j.febslet.2013.12.022 (2014).

38 Ruez, R. et al. Dendritic Cell Migration Toward CCL21 Gradient Requires Functional Cx43. Front Physiol 9, 288, doi:10.3389/fphys.2018.00288 (2018).

39 Mori, R., Power, K. T., Wang, C. M., Martin, P. \& Becker, D. L. Acute downregulation of connexin43 at wound sites leads to a reduced inflammatory response, enhanced keratinocyte proliferation and wound fibroblast migration. $J$ Cell Sci 119, 5193-5203, doi:119/24/5193 [pii]

10.1242/jcs.03320 (2006).

40 Pollok, S. et al. Connexin 43 mimetic peptide Gap27 reveals potential differences in the role of $\mathrm{Cx} 43$ in wound repair between diabetic and non-diabetic cells. J Cell Mol Med 15, 861-873, doi:10.1111/j.1582-4934.2010.01057.x (2011).

41 Ongstad, E. L., O'Quinn, M. P., Ghatnekar, G. S., Yost, M. J. \& Gourdie, R. G. A Connexin43 Mimetic Peptide Promotes Regenerative Healing and Improves 
Mechanical Properties in Skin and Heart. Advances in wound care 2, 55-62, doi:10.1089/wound.2011.0341 (2013).

42 Grek, C. L. et al. A Multicenter Randomized Controlled Trial Evaluating a Cx43-Mimetic Peptide in Cutaneous Scarring. J Invest Dermatol 137, 620-630, doi:10.1016/j.jid.2016.11.006 (2017).

43 Tarzemany, R., Jiang, G., Larjava, H. \& Hakkinen, L. Expression and function of connexin 43 in human gingival wound healing and fibroblasts. PLOS One 10, e0115524, doi:10.1371/journal.pone.0115524 (2015).

44 Zhang, Y. D. et al. Cx43- and Smad-Mediated TGF-beta/ BMP Signaling Pathway Promotes Cartilage Differentiation of Bone Marrow Mesenchymal Stem Cells and Inhibits Osteoblast Differentiation. Cell Physiol Biochem 42, 1277-1293, doi:10.1159/000478957 (2017).

45 Rossello, R. A., Wang, Z., Kizana, E., Krebsbach, P. H. \& Kohn, D. H. Connexin 43 as a signaling platform for increasing the volume and spatial distribution of regenerated tissue. Proc Natl Acad Sci $U S A$ 106, 13219-13224, doi:10.1073/pnas.0902622106 (2009).

46 Schrobback, K., Klein, T. J. \& Woodfield, T. B. The importance of connexin hemichannels during chondroprogenitor cell differentiation in hydrogel versus microtissue culture models. Tissue engineering. Part A 21, 1785-1794, doi:10.1089/ten.TEA.2014.0691 (2015).

47 Carpintero-Fernandez, P. et al. Intercellular communication via gap junction channels between chondrocytes and bone cells. Biochimica et Biophysica Acta (BBA) - Biomembranes 1860, 2499-2505, doi:10.1016/j.bbamem.2018.09.009 (2018).

48 Mayan, M. D. et al. Articular chondrocyte network mediated by gap junctions: role in metabolic cartilage homeostasis. Ann Rheum Dis 74, 275-284, doi:10.1136/annrheumdis-2013-204244 (2015).

49 Horcajada, M. N., Membrez, F. \& Offord, E. A. Compositions for use in stimulating bone growth. (2014).

50 Raederstorff, D., Richard, N., Schwager, J. \& Wertz, K. Compositions and use thereof for the treatment, co-treatment or prevention of imflammatory disorders. (2008).

51 Crea, R. Method for treatment of inflammation. US8216599B2 (2012).

52 Santiago-Mora, R., Casado-Diaz, A., De Castro, M. D. \& Quesada-Gomez, J. M. Oleuropein enhances osteoblastogenesis and inhibits adipogenesis: the effect on 
differentiation in stem cells derived from bone marrow. Osteoporosis international : a journal established as result of cooperation between the European Foundation for Osteoporosis and the National Osteoporosis Foundation of the USA 22, 675-684, doi:10.1007/s00198-010-1270-x (2011).

53 Huynh, N. P. T., Zhang, B. \& Guilak, F. High-depth transcriptomic profiling reveals the temporal gene signature of human mesenchymal stem cells during chondrogenesis. FASEB J 33, 358-372, doi:10.1096/fj.201800534R (2019).

54 Solan, J. L. \& Lampe, P. D. Spatio-temporal regulation of connexin43 phosphorylation and gap junction dynamics. Biochimica et biophysica acta. Biomembranes 1860, 83-90, doi:10.1016/j.bbamem.2017.04.008 (2018).

55 Jing, Y. et al. Chondrogenesis and osteogenesis are one continuous developmental and lineage defined biological process. Sci Rep 7, 10020, doi:10.1038/s41598-017-10048-z (2017).

56 Yang, L., Tsang, K. Y., Tang, H. C., Chan, D. \& Cheah, K. S. Hypertrophic chondrocytes can become osteoblasts and osteocytes in endochondral bone formation. Proc Natl Acad Sci $U$ S A 111, 12097-12102, doi:10.1073/pnas.1302703111 (2014).

57 Ono, N., Ono, W., Nagasawa, T. \& Kronenberg, H. M. A subset of chondrogenic cells provides early mesenchymal progenitors in growing bones. Nat Cell Biol 16, 1157-1167, doi:10.1038/ncb3067 (2014).

58 Tian, H. Detection of differentially expressed genes involved in osteoarthritis pathology. Journal of orthopaedic surgery and research 13, 49, doi:10.1186/s13018018-0734-0 (2018).

59 Karlsson, C. et al. Genome-wide expression profiling reveals new candidate genes associated with osteoarthritis. Osteoarthritis Cartilage 18, 581-592, doi:10.1016/j.joca.2009.12.002 (2010).

60 Lardy, H. A. Antibiotic inhibitors of mitochondrial energy transfer. Pharmacology \& therapeutics 11, 649-660 (1980).

61 Martin, J. A. et al. Mitochondrial electron transport and glycolysis are coupled in articular cartilage. Osteoarthritis Cartilage 20, 323-329, doi:10.1016/j.joca.2012.01.003 (2012).

62 Mayan, M. D., Blanco, F. J., Carpintero-Fernandez, P. \& Goldberg, G. S. Compositions and methods to treat inflammatory joint diseases. US Patent Application number PCT/US2014/045229 (2014). 
63 Mosteiro, L., Pantoja, C., de Martino, A. \& Serrano, M. Senescence promotes in vivo reprogramming through p16(INK)(4a) and IL-6. Aging cell 17, doi:10.1111/acel.12711 (2018).

64 Mosteiro, L. et al. Tissue damage and senescence provide critical signals for cellular reprogramming in vivo. Science 354, doi:10.1126/science.aaf4445 (2016).

65 Greene, M. A. \& Loeser, R. F. Aging-related inflammation in osteoarthritis. Osteoarthritis Cartilage 23, 1966-1971, doi:10.1016/j.joca.2015.01.008 (2015).

66 Ortiz-Montero, P., Londono-Vallejo, A. \& Vernot, J. P. Senescence-associated IL-6 and IL-8 cytokines induce a self- and cross-reinforced senescence/inflammatory milieu strengthening tumorigenic capabilities in the MCF-7 breast cancer cell line. Cell communication and signaling : CCS 15, 17, doi:10.1186/s12964-017-0172-3 (2017).

67 Moiseeva, O. et al. Metformin inhibits the senescence-associated secretory phenotype by interfering with IKK/NF-kappaB activation. Aging cell 12, 489-498, doi:10.1111/acel.12075 (2013).

68 Chien, Y. et al. Control of the senescence-associated secretory phenotype by NF-kappaB promotes senescence and enhances chemosensitivity. Genes Dev 25, 2125-2136, doi:10.1101/gad.17276711 (2011).

69 Feng, Z. et al. Oleuropein inhibits the IL-1beta-induced expression of inflammatory mediators by suppressing the activation of NF-kappaB and MAPKs in human osteoarthritis chondrocytes. Food \& function 8, 3737-3744, doi:10.1039/c7fo00823f (2017).

70 Horcajada, M. N., Membrez , F. \& Offord Cavin, E. Anti-inflammatory phytonutrients for use in the treatment or prevention of synovitis. US20160263139A1 (2013).

71 Horcajada, M. N. et al. Oleuropein or rutin consumption decreases the spontaneous development of osteoarthritis in the Hartley guinea pig. Osteoarthritis Cartilage 23, 94-102, doi:10.1016/j.joca.2014.08.016 (2015).

72 Horcajada, M. N., Membrez , F. \& Offord Cavin, E. Compositions for use in cartilage breakdown. US20160120891A1 (2013).

73 Takeda, R., Koike, T., Taniguchi, I. \& Tanaka, K. Double-blind placebocontrolled trial of hydroxytyrosol of Olea europaea on pain in gonarthrosis. Phytomedicine : international journal of phytotherapy and phytopharmacology 20 , 861-864, doi:10.1016/j.phymed.2013.03.021 (2013). 
74 Psaltopoulou, T., Kosti, R. I., Haidopoulos, D., Dimopoulos, M. \& Panagiotakos, D. B. Olive oil intake is inversely related to cancer prevalence: a systematic review and a meta-analysis of 13,800 patients and 23,340 controls in 19 observational studies. Lipids in health and disease 10, 127, doi:10.1186/1476-511X10-127 (2011).

75 Pazoki-Toroudi, H. et al. Targeting mTOR signaling by polyphenols: A new therapeutic target for ageing. Ageing research reviews 31, 55-66, doi:10.1016/j.arr.2016.07.004 (2016).

76 Rigacci, S. \& Stefani, M. Nutraceutical Properties of Olive Oil Polyphenols. An Itinerary from Cultured Cells through Animal Models to Humans. International journal of molecular sciences 17, doi:10.3390/ijms17060843 (2016).

77 Fujita, K. et al. Cinnamtannin B-1 Promotes Migration of Mesenchymal Stem Cells and Accelerates Wound Healing in Mice. PLoS One 10, e0144166, doi:10.1371/journal.pone.0144166 (2015).

78 Pastore, S. et al. Plant polyphenols regulate chemokine expression and tissue repair in human keratinocytes through interaction with cytoplasmic and nuclear components of epidermal growth factor receptor system. Antioxid Redox Signal 16, 314-328, doi:10.1089/ars.2011.4053 (2012).

79 Studzinska-Sroka, E. et al. Anti-inflammatory Activity and Phytochemical Profile of Galinsoga Parviflora Cav. Molecules 23, doi:10.3390/molecules23092133 (2018).

80 Chen, Y. et al. Activation of Nrf2 Attenuates Pulmonary Vascular Remodeling via Inhibiting Endothelial-to-Mesenchymal Transition: an Insight from a Plant Polyphenol. International journal of biological sciences 13, 1067-1081, doi:10.7150/ijbs.20316 (2017).

81 Parmar, K. M., Shende, P. R., Katare, N., Dhobi, M. \& Prasad, S. K. Wound healing potential of Solanum xanthocarpum in streptozotocin-induced diabetic rats. $J$ Pharm Pharmacol 70, 1389-1400, doi:10.1111/jphp.12975 (2018).

82 Omar, S. H. Oleuropein in olive and its pharmacological effects. Scientia pharmaceutica 78, 133-154, doi:10.3797/scipharm.0912-18 (2010).

83 Barbaro, B. et al. Effects of the olive-derived polyphenol oleuropein on human health. International journal of molecular sciences 15, 18508-18524, doi:10.3390/ijms151018508 (2014). 
84 Angelino, D. et al. Chemical and cellular antioxidant activity of phytochemicals purified from olive mill waste waters. Journal of agricultural and food chemistry 59, 2011-2018, doi:10.1021/jf103881b (2011).

85 Sarsour, E. H. et al. MnSOD activity regulates hydroxytyrosol-induced extension of chronological lifespan. Age 34, 95-109, doi:10.1007/s11357-011-9223-7 (2012).

86 Omar, S. H. Cardioprotective and neuroprotective roles of oleuropein in olive. Saudi pharmaceutical journal : SPJ : the official publication of the Saudi Pharmaceutical Society 18, 111-121, doi:10.1016/j.jsps.2010.05.005 (2010).

87 Valenzuela, R. et al. Molecular adaptations underlying the beneficial effects of hydroxytyrosol in the pathogenic alterations induced by a high-fat diet in mouse liver: PPAR-alpha and Nrf2 activation, and NF-kappaB down-regulation. Food \& function 8, 1526-1537, doi:10.1039/c7fo00090a (2017).

88 St-Laurent-Thibault, C., Arseneault, M., Longpre, F. \& Ramassamy, C. Tyrosol and hydroxytyrosol, two main components of olive oil, protect N2a cells against amyloid-beta-induced toxicity. Involvement of the NF-kappaB signaling. Current Alzheimer research 8, 543-551 (2011).

89 Yonezawa, Y. et al. Anti-inflammatory effects of olive-derived hydroxytyrosol on lipopolysaccharide-induced inflammation in RAW264.7 cells. The Journal of veterinary medical science 80, 1801-1807, doi:10.1292/jvms.18-0250 (2018).

90 Wang, W. et al. Hydroxytyrosol NO regulates oxidative stress and NO production through SIRT1 in diabetic mice and vascular endothelial cells. Phytomedicine : international journal of phytotherapy and phytopharmacology $\mathbf{5 2}$, 206-215, doi:10.1016/j.phymed.2018.09.208 (2019).

91 Visioli, F., Bellomo, G. \& Galli, C. Free radical-scavenging properties of olive oil polyphenols. Biochem Biophys Res Commun 247, 60-64, doi:10.1006/bbrc.1998.8735 (1998).

92 Andreadou, I. et al. Acute doxorubicin cardiotoxicity is successfully treated with the phytochemical oleuropein through suppression of oxidative and nitrosative stress. J Mol Cell Cardiol 42, 549-558, doi:10.1016/j.yjmcc.2006.11.016 (2007).

93 Rigacci, S. et al. Oleuropein aglycone induces autophagy via the AMPK/mTOR signalling pathway: a mechanistic insight. Oncotarget 6, 35344-35357, doi:10.18632/oncotarget.6119 (2015). 
94 Wang, W. et al. Hydroxytyrosol regulates the autophagy of vascular adventitial fibroblasts through the SIRT1-mediated signaling pathway. Can J Physiol Pharmacol 96, 88-96, doi:10.1139/cjpp-2016-0676 (2018).

95 Cetrullo, S., D'Adamo, S., Guidotti, S., Borzi, R. M. \& Flamigni, F. Hydroxytyrosol prevents chondrocyte death under oxidative stress by inducing autophagy through sirtuin 1-dependent and -independent mechanisms. Biochim Biophys Acta 1860, 1181-1191, doi:10.1016/j.bbagen.2016.03.002 (2016).

96 Kim, Y., Choi, Y. \& Park, T. Hepatoprotective effect of oleuropein in mice: mechanisms uncovered by gene expression profiling. Biotechnology journal 5, 950960, doi:10.1002/biot.201000068 (2010).

97 Jemai, H., El Feki, A. \& Sayadi, S. Antidiabetic and antioxidant effects of hydroxytyrosol and oleuropein from olive leaves in alloxan-diabetic rats. J Agric Food Chem 57, 8798-8804, doi:10.1021/jf901280r (2009).

98 Bulotta, S. et al. Beneficial effects of the olive oil phenolic components oleuropein and hydroxytyrosol: focus on protection against cardiovascular and metabolic diseases. Journal of translational medicine 12, 219, doi:10.1186/s12967014-0219-9 (2014).

$99 \mathrm{Li}, \mathrm{C}$. J. et al. MicroRNA-188 regulates age-related switch between osteoblast and adipocyte differentiation. J Clin Invest 125, 1509-1522, doi:10.1172/JCI77716 (2015).

100 Moerman, E. J., Teng, K., Lipschitz, D. A. \& Lecka-Czernik, B. Aging activates adipogenic and suppresses osteogenic programs in mesenchymal marrow stroma/stem cells: the role of PPAR-gamma2 transcription factor and TGF-beta/BMP signaling pathways. Aging cell 3, 379-389, doi:10.1111/j.1474-9728.2004.00127.x (2004).

101 Ambrosi, T. H. et al. Adipocyte Accumulation in the Bone Marrow during Obesity and Aging Impairs Stem Cell-Based Hematopoietic and Bone Regeneration. Cell stem cell 20, 771-784 e776, doi:10.1016/j.stem.2017.02.009 (2017).

102 Solan, J. L. \& Lampe, P. D. Specific Cx43 phosphorylation events regulate gap junction turnover in vivo. FEBS Lett 588, 1423-1429, doi:10.1016/j.febslet.2014.01.049 (2014).

103 Thevenin, A. F., Margraf, R. A., Fisher, C. G., Kells-Andrews, R. M. \& Falk, M. M. Phosphorylation regulates connexin43/ZO-1 binding and release, an important step in gap junction turnover. Mol Biol Cell 28, 3595-3608, doi:10.1091/mbc.E16-070496 (2017). 
104 Spagnol, G. et al. Connexin43 Carboxyl-Terminal Domain Directly Interacts with beta-Catenin. Int J Mol Sci 19, doi:10.3390/ijms19061562 (2018).

105 Giepmans, B. N., Verlaan, I. \& Moolenaar, W. H. Connexin-43 interactions with ZO-1 and alpha- and beta-tubulin. Cell Commun Adhes 8, 219-223 (2001).

106 Gonzalez-Sanchez, A. et al. Connexin43 recruits PTEN and Csk to inhibit cSrc activity in glioma cells and astrocytes. Oncotarget 7, 49819-49833, doi:10.18632/oncotarget.10454 (2016).

107 Kotini, M. et al. Gap junction protein Connexin-43 is a direct transcriptional regulator of $\mathrm{N}$-cadherin in vivo. Nature communications 9, 3846, doi:10.1038/s41467018-06368-x (2018).

108 Price, J. S. et al. The role of chondrocyte senescence in osteoarthritis. Aging cell 1, 57-65 (2002).

109 Gao, S. G. et al. Correlation between senescence-associated betagalactosidase expression in articular cartilage and disease severity of patients with knee osteoarthritis. International journal of rheumatic diseases 19, 226-232, doi:10.1111/1756-185X.12096 (2016).

110 Kuilman, T. et al. Oncogene-induced senescence relayed by an interleukindependent inflammatory network. Cell 133, 1019-1031, doi:10.1016/j.cell.2008.03.039 (2008).

111 Acosta, J. C. et al. Chemokine signaling via the CXCR2 receptor reinforces senescence. Cell 133, 1006-1018, doi:10.1016/j.cell.2008.03.038 (2008).

112 Jing, $H$. et al. Opposing roles of NF-kappaB in anti-cancer treatment outcome unveiled by cross-species investigations. Genes Dev 25, 2137-2146, doi:10.1101/gad.17620611 (2011).

113 Acosta, J. C. et al. A complex secretory program orchestrated by the inflammasome controls paracrine senescence. Nat Cell Biol 15, 978-990, doi:10.1038/ncb2784 (2013).

114 Hubackova, S., Krejcikova, K., Bartek, J. \& Hodny, Z. IL1- and TGFbeta-Nox4 signaling, oxidative stress and DNA damage response are shared features of replicative, oncogene-induced, and drug-induced paracrine 'bystander senescence'. Aging 4, 932-951, doi:10.18632/aging.100520 (2012).

115 Orjalo, A. V., Bhaumik, D., Gengler, B. K., Scott, G. K. \& Campisi, J. Cell surface-bound IL-1alpha is an upstream regulator of the senescence-associated IL6/IL-8 cytokine network. Proc Natl Acad Sci $U S A$ 106, 17031-17036, doi:10.1073/pnas.0905299106 (2009). 
116 Demaria, M. et al. An essential role for senescent cells in optimal wound healing through secretion of PDGF-AA. Developmental cell 31, 722-733, doi:10.1016/j.devcel.2014.11.012 (2014).

117 Jun, J. I. \& Lau, L. F. The matricellular protein CCN1 induces fibroblast senescence and restricts fibrosis in cutaneous wound healing. Nat Cell Biol 12, 676685, doi:10.1038/ncb2070 (2010).

118 Munoz-Espin, D. \& Serrano, M. Cellular senescence: from physiology to pathology. Nat Rev Mol Cell Biol 15, 482-496, doi:10.1038/nrm3823 (2014).

119 Castejon, M. L. et al. Oleuropein down-regulated IL-1beta-induced inflammation and oxidative stress in human synovial fibroblast cell line SW982. Food \& function 8, 1890-1898, doi:10.1039/c7fo00210f (2017).

120 Raptis, L. H., Brownell, H. L., Firth, K. L. \& Mackenzie, L. W. A novel technique for the study of intercellular, junctional communication: electroporation of adherent cells on a partly conductive slide. DNA Cell Biol 13, 963-975 (1994).

121 Raptis, L. et al. Electroporation of adherent cells in situ for the study of signal transduction and gap junctional communication. Methods Mol Biol 423, 173-189, doi:10.1007/978-1-59745-194-9_12 (2008). 


\section{Figures}

a

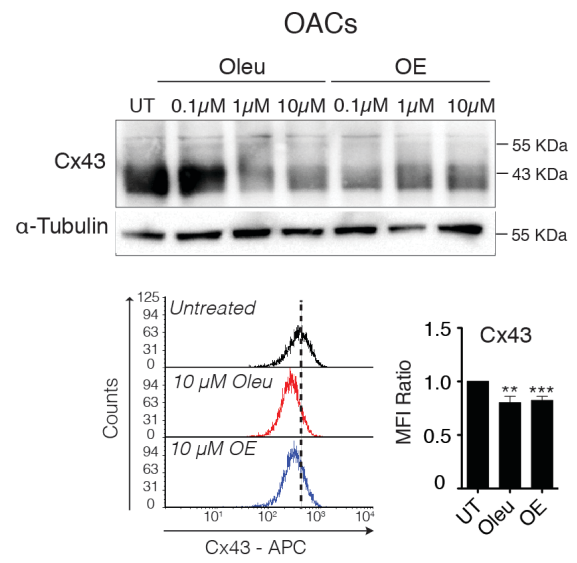

b

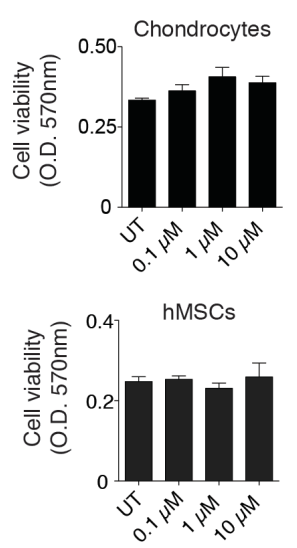

C
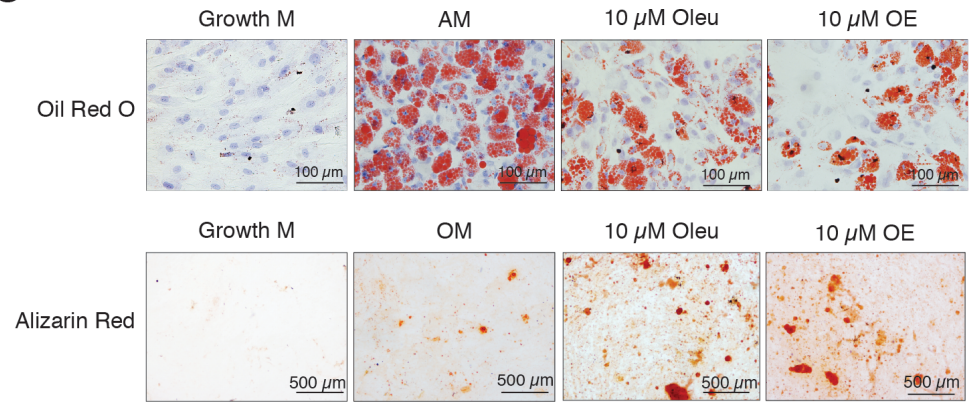

d

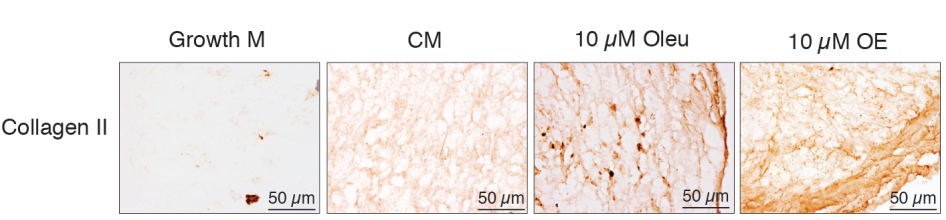

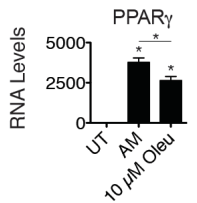
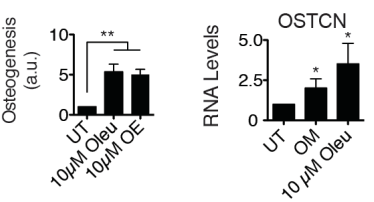

e

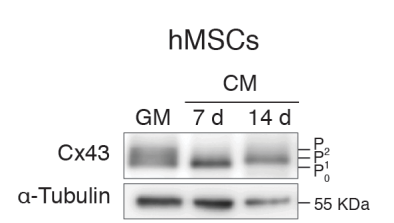

f

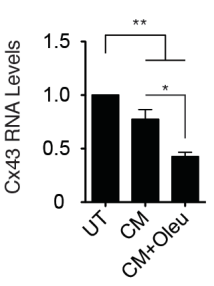

g

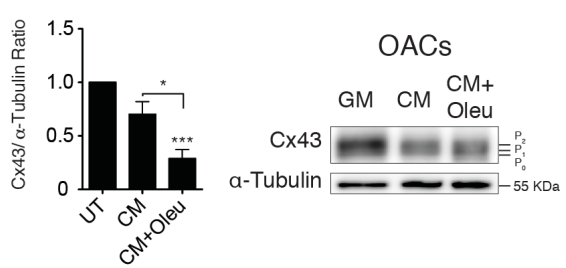

Figure 1. Downregulation of $\mathrm{Cx} 43$ during chondrogenesis improves differentiation towards chondrocytes. (a) Treatment of OACs with oleuropein (Oleu) or olive extract (OE) for $2 \mathrm{~h}$ significantly downregulates $\mathrm{Cx} 43$ protein (western blot and flow cytometry assays). The graph from the flow cytometry assays represents the median fluorescence intensity $\left(n=9\right.$; mean \pm s.e.m.; ${ }^{\star \star} P<0.01$, ${ }^{* \star *} P<0.0001$; Mann-Whitney test). (b) Cell viability measured by MTT assay of hMSCs $(n=5)$ and chondrocytes $(n=7)$ exposed to different concentrations of oleuropein (Oleu) for $17 \mathrm{~h}$ (mean \pm s.e.m.). (c) Differentiation capacity of hMSCs 
grown in adipogenic (top, 21 days) or osteogenic (bottom, 21 days) medium supplemented with $10 \mu \mathrm{M}$ Oleu or $10 \mu \mathrm{M}$ OE. hMSCs cultured in growth medium were used as a control. Top, adipogenic evaluation by oil red $\mathrm{O}$ for lipid staining and by PPARY gene expression ( $n=4-5$; mean \pm s.e.m.; ${ }^{*} P<0.05$; Mann-Whitney test). Oil red $\mathrm{O}$ quantification represents the ratio of cells containing lipid deposits to the total number of cells $(n=10-13$ images from 2 independent experiments; mean \pm s.e.m. ; ${ }^{* \star *} P<0.0001$; Mann-Whitney test). Values were normalized to hMSCs differentiated in adipogenic medium without treatment (UT). Alizarin red staining was used to detect calcium deposits for osteogenic differentiation. Values were obtained by counting red pixels and normalized to those of hMSCs differentiated in osteogenic medium without treatment (UT) ( $\mathrm{n}=4-6$; mean \pm s.e.m.; ${ }^{\star} P<0.05,{ }^{* \star} P<0.01$; MannWhitney test). OSTCN gene expression was measured to confirm osteogenic differentiation ( $\mathrm{n}=4$; mean \pm s.e.m.; ${ }^{\star} P<0.05$; Mann-Whitney test). (d) Differentiation capacity of hMSCs grown in chondrogenic medium as micromasses for 30 days. Representative immunohistochemical images for Col2A1 indicate chondrogenic differentiation in micromasses. The quantification is shown on the right $(n=4-6$; mean \pm s.e.m.; * $P<0.05$; Mann-Whitney test). Chondrogenesis was also evaluated by ACAN gene expression quantification $\left(n=3-4\right.$; mean \pm s.e.m.; ${ }^{*} P<0.05$; Mann-Whitney test). (e) Cx43 protein levels in hMSCs differentiated for 7 and 14 days in the presence of chondrogenic medium (CM) in comparison to untreated hMSCs cultured in normal growth medium (GM). (f) Cx43 RNA expression of hMSCs cultured for 14 days in the presence of chondrogenic medium (CM) alone or supplemented with 10 $\mu \mathrm{M}$ oleuropein (Oleu). Data were normalized to HPRT-1 levels $(n=5-6$; mean \pm s.e.m. ${ }^{\star} P<0.05,{ }^{\star \star} P<0.01$; Mann-Whitney test). (g) Cx43 protein levels were analysed by western blot in OACs differentiated for 7 days in the presence of chondrogenic medium (CM), supplemented with $10 \mu \mathrm{M}$ oleuropein (Oleu). The graph represents the quantification from three independent experiments (mean \pm s.e.m.; ${ }^{*} P<0.05$, ${ }^{\star \star *} P<0.0001$; Student's $t$ test). 


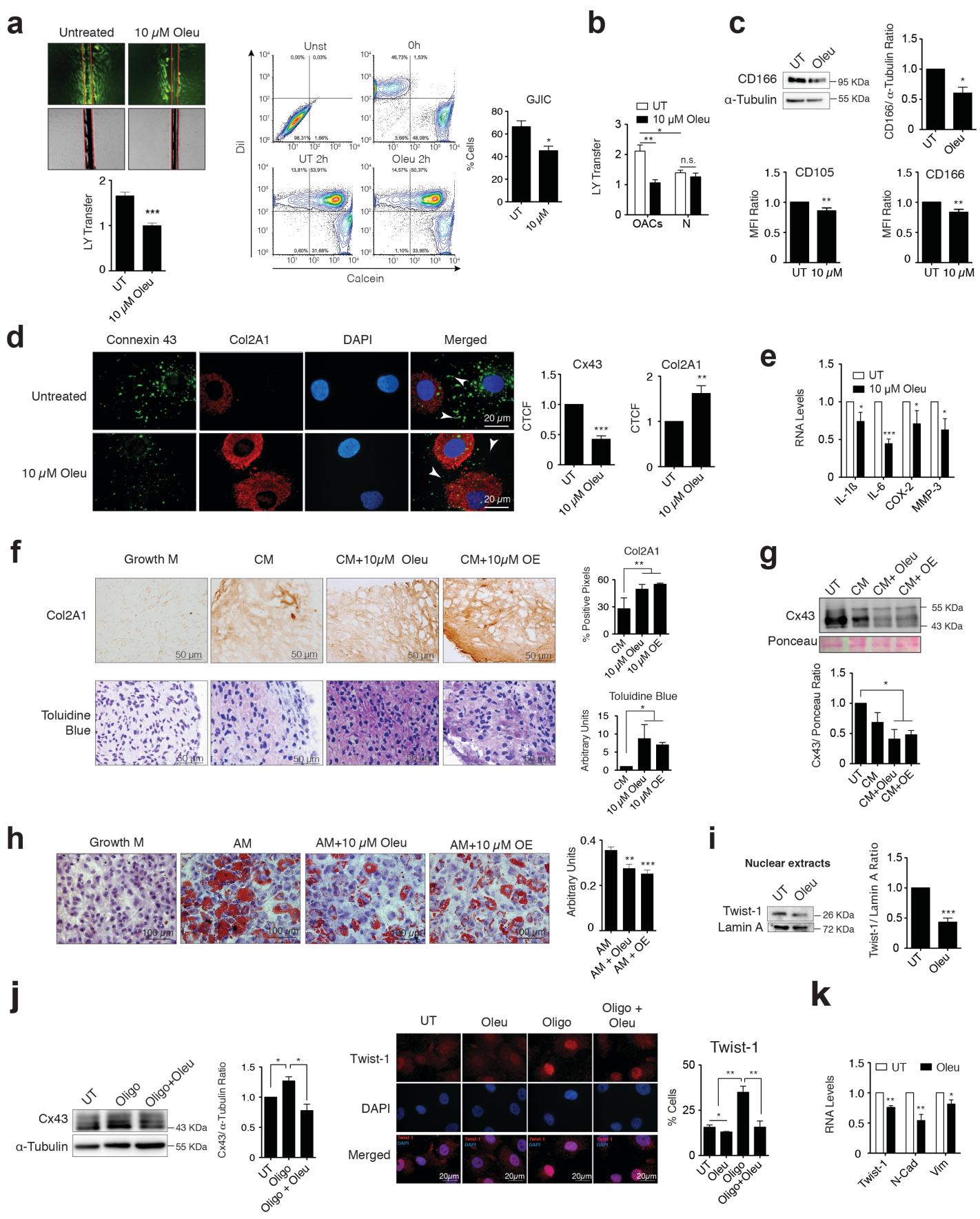

Figure 2. Downregulation of $\mathrm{Cx43}$ by oleuropein restores chondrocyte redifferentiation in chondrocytes from patients with OA. (a) Oleuropein treatment significantly decreases GJIC evaluated by an SL/DT assay when OACs were exposed with this molecule for $2 \mathrm{~h}$ (top, $\mathrm{n}=6$; mean \pm s.e.m.; ${ }^{* * *} P<0.0001$; MannWhitney test). The results were confirmed by calcein transfer and flow cytometry ( $\mathrm{n}=4$, mean \pm s.e.m.; ${ }^{*} P<0.05$; Mann-Whitney test). (b) Graph showing the effect of Oleu on GJIC when healthy chondrocytes $(\mathrm{N})$ are exposed to $10 \mu \mathrm{M}$ Oleu compared with OACs ( $\mathrm{n}=3$; mean \pm s.e.m.; ${ }^{*} P<0.05,{ }^{* *} P<0.01$; Mann-Whitney test). (c) OACs cultured for 7 days in DMEM $10 \%$ (UT) show reduced expression of the 
mesenchymal markers CD105 and CD166 when supplemented with $10 \mu \mathrm{M}$ Oleu. Protein levels were analysed by flow cytometry $\left(n=7\right.$; mean \pm s.e.m.; ${ }^{*} P<0.05$, ${ }^{\star \star} P<0.01$; Mann-Whitney test. In addition, CD166 levels were analysed by western blot ( $\mathrm{n}=3$; mean \pm S.e.m.; ${ }^{*} P<0.05$; Student's $t$ test). (d) Downregulation of $\mathrm{Cx} 43$ (green) by Oleu upregulates the main articular chondrocyte marker Col2A1 (red), as detected by immunofluorescence in OACs treated with Oleu or OE. Nuclei were stained with DAPI. Graphs represent the corrected total cell fluorescence (CTCF) measured with ImageJ with $>8$ images per condition. Data represent mean \pm s.e.m ( ${ }^{* *} P<0.01,{ }^{* *} P<0.0001$; Mann-Whitney test). (e) mRNA levels of IL-1B, IL-6, COX-2 and MMP-3 of OACs cultured in normal medium (UT, DMEM 10\% FBS) exposed to $10 \mu \mathrm{M}$ Oleu for $2 \mathrm{~h}$ ( $\mathrm{n}=3-7$; mean \pm s.e.m.; ${ }^{\star} P<0.05,{ }^{* \star *} P<0.0001$; Mann-Whitney test). (f) Immunohistochemistry (Col2A1) and toluidine blue staining (proteoglycan subunits) indicate significant enrichment in cartilage matrix components in OACs micromasses grown in $3 D$ culture for 30 days in chondrogenic medium (CM) when supplemented with $10 \mu \mathrm{M}$ Oleu or OE ( $\mathrm{n}=4-6$ (Col2A1); $\mathrm{n}=6-7$ (toluidine blue); mean \pm s.e.m.; ${ }^{\star} P<0.05 ;{ }^{\star \star} P<0,01$; Mann-Whitney test). (g) Cx43 levels detected by western blot (normalized to Ponceau staining) are reduced when OACs micromasses are exposed to chondrogenic medium (CM) supplemented with $10 \mu \mathrm{M}$ Oleu or OE for 21 days. ( $\mathrm{n}=3-4$; mean \pm s.e.m.; ${ }^{*} P<0.05$; Mann-Whitney test). (h) Oil red staining showing reduced OACs dedifferentiation upon exposure to Oleu or OE in adipogenic medium. The graph represents the ratio of cells containing lipid deposits to the total number of cells $\left(\mathrm{n}=20\right.$ images; mean \pm s.e.m. ${ }^{* \star} P<0.01,{ }^{* *} P<0.0001$; Mann-Whitney test). (i) Nuclear levels of Twist-1 were decreased in OACs cultured with $10 \mu \mathrm{M}$ Oleu for 2 hours. Lamin A was used as a loading control ( $n=3$; mean \pm s.e.m, ${ }^{\star \star \star} P<0.0001$, Student's $t$ test). (j) Twist-1 (red) activation measured by immunofluorescence to detect nuclear translocation when primary OACs were treated with $5 \mu \mathrm{g} / \mathrm{ml}$ oligomycin for $1 \mathrm{~h}$ is partially abrogated by $\mathrm{Cx} 43$ downregulation and $10 \mu \mathrm{M}$ Oleu. Nuclei were stained with DAPI. The graph represents the percentage of cells with Twist-1 nuclear localization ( $\mathrm{n}=4-5$; mean \pm s.e.m, ${ }^{*} P<0.05,{ }^{* \star} P<0.01$; Mann-Whitney test). Scale bar, $20 \mu \mathrm{m}$. On the right, western blot $(\mathrm{n}=4)$ to show the effects of $1-\mathrm{h}$ treatment with Oleu or oligomycin on $\mathrm{Cx} 43$ protein levels in primary OACs. Quantification is shown on the right (mean \pm s.e.m.; ${ }^{*} P<0.05$; Mann-Whitney test). (k) The RNA expression of the EMT markers Twist-1, N-Cadherin and Vimentin was upregulated in OACs treated with $10 \mu \mathrm{M}$ Oleu for 2 hours. Data were normalized to HPRT-1 levels ( $n=5-6$, mean \pm s.e.m, ${ }^{\star} P<0.05,{ }^{\star \star} P<0.01$; Mann-Whitney test). 

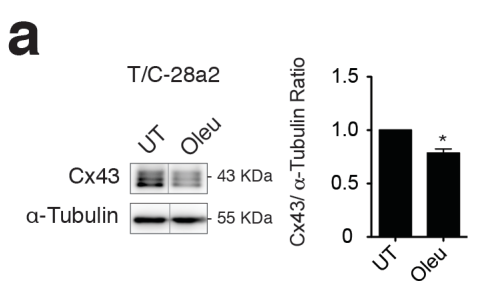

b
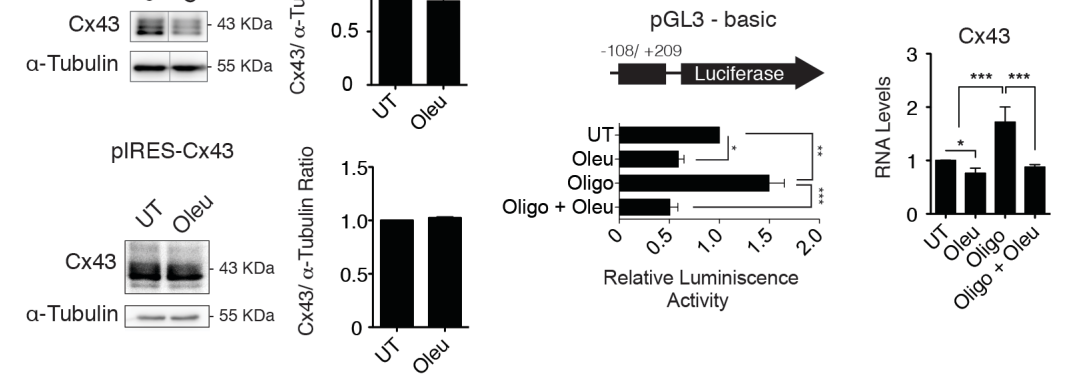

C
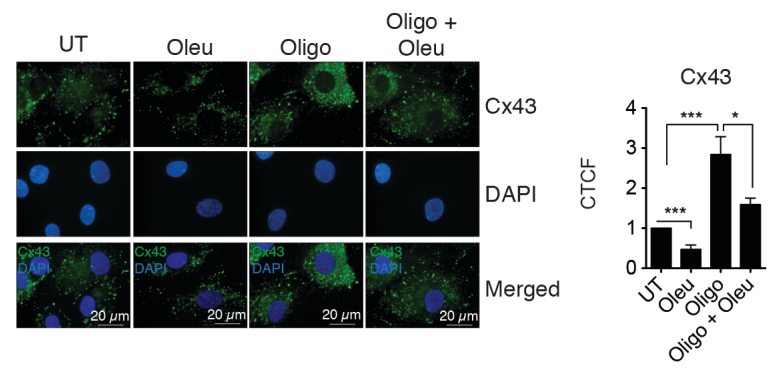

Figure 3. Oleuropein modulates the Cx43 promoter activity in chondrocytes.

(a) Treatment with $10 \mu \mathrm{M}$ oleuropein (Oleu) for $2 \mathrm{~h}$ decreases $\mathrm{Cx} 43$ protein levels in T/C-28a2 cells $(n=4)$, but this effect is not observed in the same cell line overexpressing Cx43 (pIRES-Cx43) ( $\mathrm{n}=2$, mean \pm s.e.m. ${ }^{*} P<0.05$; Mann-Whitney test). (b) Luciferase reporter assay indicating that Oleu inhibits Cx43 promoter activity. The graphs indicate the normalized luminescence activity in the T/C-28a2 chondrocyte cell line transfected with a pGL3-basic plasmid containing 300 base pairs of $\mathrm{Cx} 43$ promoter ligated to the luciferase gene. Cells were cultured in DMEM with $10 \%$ FBS (UT) and with $5 \mu \mathrm{g} / \mathrm{ml}$ oligomycin or $10 \mu \mathrm{M}$ Oleu for $1 \mathrm{~h}$ as indicated ( $\mathrm{n}=6$; mean \pm s.e.m.; ${ }^{* *} P<0.01$; Mann-Whitney test). On the right, Oleu reduces $\mathrm{Cx} 43$ gene expression and abolishes oligomycin-induced upregulation of Cx43 mRNA in OACs treated for $1 \mathrm{~h}$ following cell seeding ( $\mathrm{n}=2-4$; mean \pm s.e.m.; ${ }^{*} P<0.05$; ${ }^{* * *} P<$ 0.0001 ; Student's $t$ test). (c) The effects of 1-h treatment with Oleu or oligomycin on Cx43 protein levels in primary OACs were confirmed by immunofluorescence $(n=9$, mean \pm s.e.m.; ${ }^{\star} P<0.05 ;{ }^{\star \star \star} P<0.0001$; Mann-Whitney test). 
a

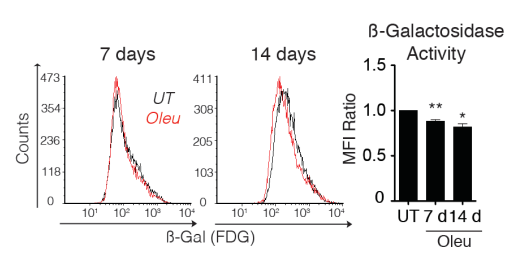

b

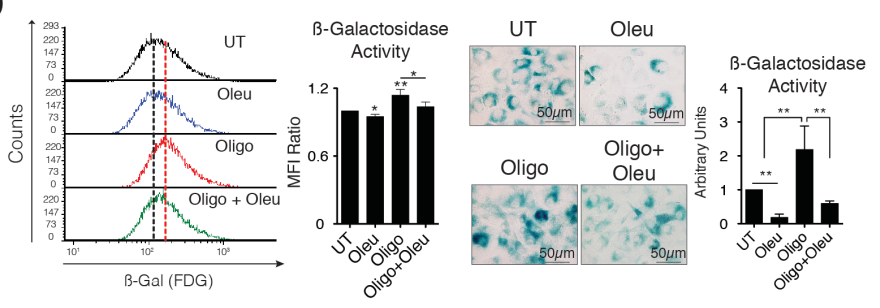

e

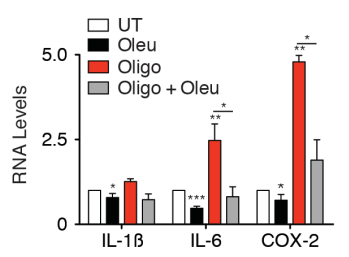

$\mathbf{f}$

C

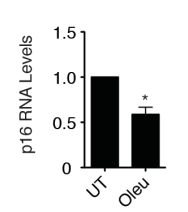

d
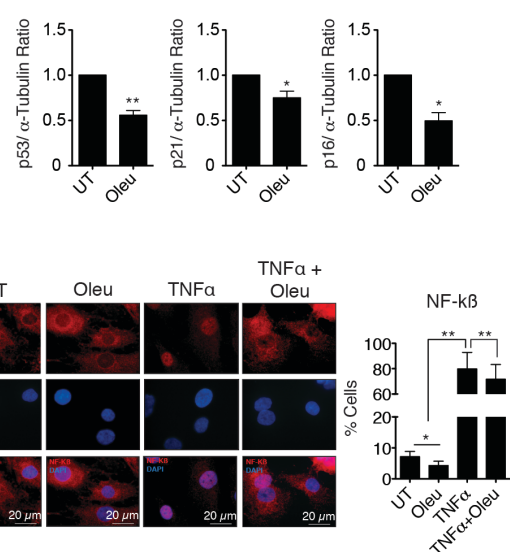

i

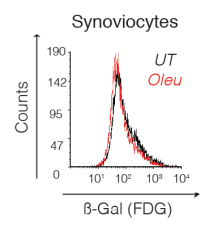

m

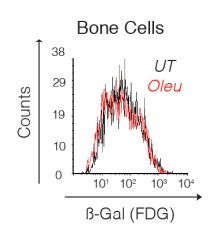

9

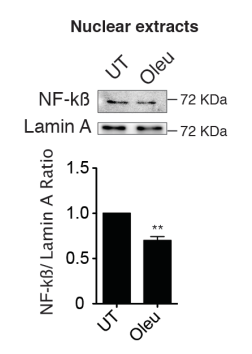

k

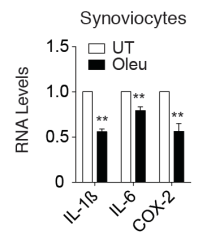

$\mathbf{n}$

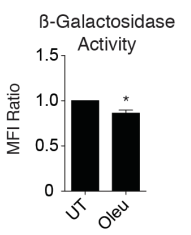

j
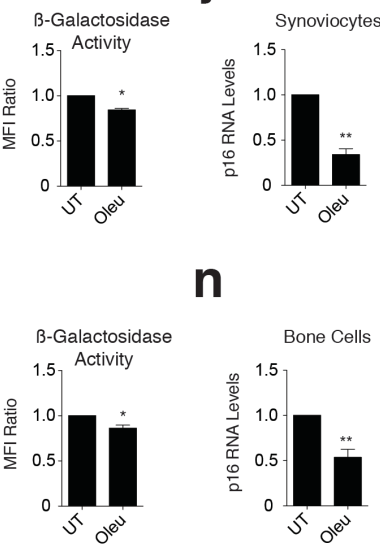

0

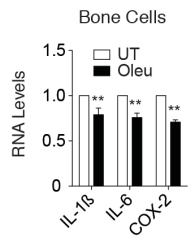

Figure 4. Cx43 downregulation by oleuropein decreased chondrocyte senescence. (a) Oleuropein (Oleu) treatment, which reduces Cx43 protein in OACs (See Fig. 1a), also reduces senescence as detected by SA $B G$ and flow cytometry. Cells were treated with $10 \mu \mathrm{M}$ Oleu for 7 and 14 days $\left(\mathrm{n}=3-7\right.$; mean \pm s.e.m.; ${ }^{*} P<0.05$, ${ }^{\star \star} P<0.01$; Mann-Whitney test). (b) Downregulation of $\mathrm{Cx} 43$ by Oleu treatment reduces senescence and abolishes oligomycin-induced senescence. The graphs show the comparative analysis of SABG activity measured by flow cytometry of OACs exposed for $24 \mathrm{~h}$ to Oleu or oligomycin as indicated $(n=5-6$; mean \pm s.e.m.; ${ }^{\star} P<0.05,{ }^{\star \star} P<0.01$; Mann-Whitney test). Right, to further confirm these results, SABG activity, determined by X-Gal cleavage and cell staining (blue), was evaluated by microscopy in OACs treated for 7 days with these drugs. The images are representative from three independent experiments and quantification is shown on 
the right (mean \pm s.e.m.; ${ }^{\star} P<0.05,{ }^{\star \star} P<0.01,{ }^{\star \star \star} P<0.0001$; Mann-Whitney test). (c) p16 mRNA expression of OACs treated with $10 \mu \mathrm{M}$ Oleu for $2 \mathrm{~h}$. Data were normalized to HPRT-1 levels ( $\mathrm{n}=5$, mean \pm s.e.m, ${ }^{*} P<0.05$; Mann-Whitney test). (d) Western blot of p53, p21 and p16 levels in OACs treated with $10 \mu \mathrm{M}$ oleuropein (Oleu) for 2 h. a-tubulin was used as a loading control ( $\mathrm{n}=2-3$; mean \pm s.e.m.; ${ }^{*} P<0.05$, ${ }^{\star \star} P<0.01$; Student's $t$ test). (e) Downregulation of $\mathrm{C} \times 43$ by Oleu attenuates the IL-6 and COX-2 upregulation when OACs are exposed to oligomycin for $1 \mathrm{~h}(\mathrm{n}=3-9$; mean \pm s.e.m.; ${ }^{*} P<0.05,{ }^{* *} P<0.01,{ }^{* *} P<0.0001$; Mann-Whitney test). (f) Western blot $(n=3)$ shows the effect of Oleu and TNFa treatments on Cx43 protein levels in primary OACs (mean \pm s.e.m.; ${ }^{\star} P<0.05,{ }^{\star \star} P<0.01$; Student's $t$ test). On the right, NF$\mathrm{KB}$ (red) detected by immunofluorescence in OACs treated with TNFa to induce NF$\mathrm{KB}$ nuclear translocation and activation. This effect is partially abolished by $1-\mathrm{h}$ Oleu treatment. Nuclei were stained with DAPI. The graph represents the cell percentage with nuclear NF-kB staining ( $\mathrm{n}=5$; mean \pm S.e.m.; ${ }^{\star} P<0.05$; Mann-Whitney test). (Pending WB) (g) Nuclear levels of NF-kß were decreased in OACs cultured with 10 $\mu \mathrm{M}$ Oleu for 2 hours. Lamin $\mathrm{A}$ was used as a loading control $(\mathrm{n}=3$; mean \pm s.e.m, ${ }^{\star \star} P<0.01$, Student's $t$ test). (h) $\mathrm{C} \times 43$ protein levels analysed by western blot in synoviocytes treated with oleuropein (Oleu) for 2 hours. a-tubulin was used as a loading control ( $\mathrm{n}=7$; mean \pm S.e.m.; ${ }^{\star} P<0.05$; Mann-Whitney test). (i) Treatment of synoviocytes with $10 \mu \mathrm{M}$ of oleuropein (Oleu) for 7 days reduces cell senescence, as detected by SABG and flow cytometry $\left(n=4\right.$; mean \pm S.e.m.; ${ }^{*} P<0.05$; Mann-Whitney test). (j) p16 mRNA levels of synoviocytes treated with $10 \mu \mathrm{M}$ Oleu for $2 \mathrm{~h}$. Data were normalized to HPRT-1 levels $\left(\mathrm{n}=4\right.$, mean \pm s.e.m, ${ }^{\star \star} P<0.01$; Student's $t$ test). (k) mRNA levels of IL-1B, IL-6 and COX-2 of synoviocytes cultured in normal medium (UT, DMEM 10\% FBS) exposed to $10 \mu \mathrm{M}$ Oleu for $2 \mathrm{~h}$. Data were normalized to HPRT-1 levels ( $\mathrm{n}=4$; mean \pm s.e.m.; ${ }^{* *} P<0.01$; Student's $t$ test). (I) $\mathrm{Cx} 43$ protein levels analysed by western blot in bone cells treated with oleuropein (Oleu) for 2 hours. atubulin was used as a loading control $\left(n=4\right.$; mean \pm s.e.m.; ${ }^{*} P<0.05$; Mann-Whitney test). (m) $10 \mu \mathrm{M}$ of oleuropein (Oleu) treatment for 7 days reduces senescence levels in bone cells as detected by SA $\beta G$ and flow cytometry $\left(n=3\right.$; mean \pm s.e.m.; ${ }^{*} P<0.05$; Student's $t$ test). (n) p16 mRNA expression of bone cells treated with $10 \mu \mathrm{M}$ Oleu for 2 h. Data were normalized to HPRT-1 levels $\left(n=4\right.$, mean \pm s.e.m, ${ }^{* \star} P<0.01$; Student's $t$ test). (o) mRNA levels of IL-1B, IL- 6 and COX-2 of bone cells cultured in normal medium (UT, DMEM 10\% FBS) exposed to $10 \mu \mathrm{M}$ Oleu for $2 \mathrm{~h}$. Data were normalized to HPRT-1 levels ( $n=3-4$; mean \pm s.e.m.; ${ }^{* \star} P<0.01$; Student's $t$ test). 
Context of damaged tissue: Cx43 overactivity in osteoarthritis

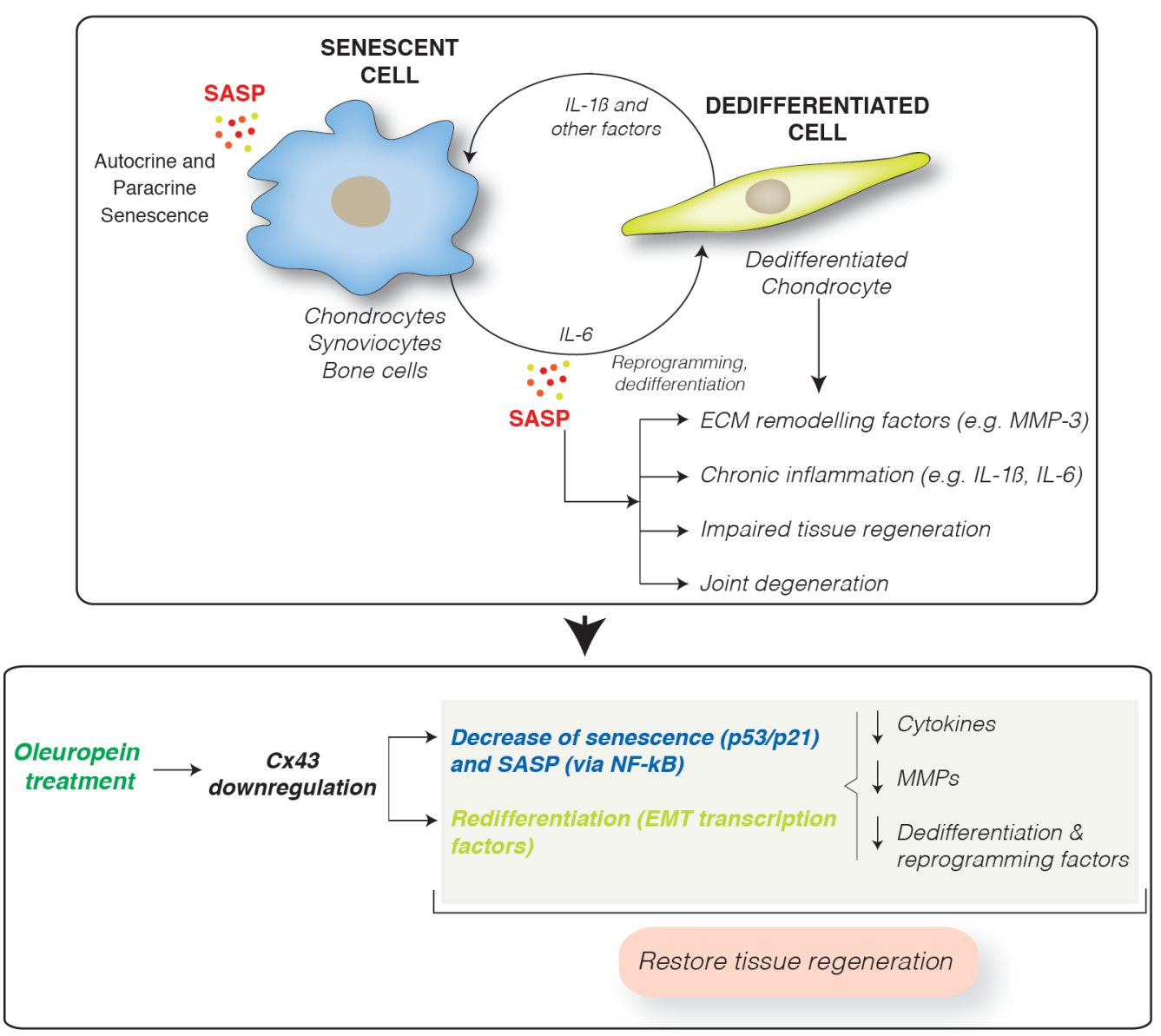

Figure 5. Cx43 overexpression leads to accumulation of dedifferentiated and senescent cells involved in disease progression in OA patients. These phenotypic changes results in the synthesis of ECM remodelling factors involved in tissue degradation (MMPs) and proinflammatory factors, such as IL-1B and IL-6, which facilitate the dedifferentiation and reprogramming of neighbouring cells. These factors may further spread senescence and dedifferentiation to surrounding tissues contributing to joint degeneration. Downregulation of $\mathrm{Cx} 43$ by oleuropein treatment contributes to the elimination of senescent cells and redifferentiation of osteoarthritic chondrocytes into fully differentiated cells, able to support the ECM composition and restoring the regenerative capacity of the tissue. 
bioRxiv preprint doi: https://doi.org/10.1101/686535; this version posted June 28,2019 . The copyright holder for this preprint (which was

not certified by peer review) is the author/funder. All rights reserved. No reuse allowed without permission. 